\title{
Driven cofactor systems and Hamilton-Jacobi separability
}

\author{
W. Sarlet ${ }^{a, b}$ and G. Waeyaert ${ }^{a}$ \\ ${ }^{a}$ Department of Mathematics, Ghent University \\ Krijgslaan 281, B-9000 Ghent, Belgium \\ ${ }^{b}$ Department of Mathematics and Statistics, La Trobe University \\ Bundoora, Victoria 3086, Australia
}

\begin{abstract}
This is a continuation of the work initiated in [18] on so-called driven cofactor systems, which are partially decoupling second-order differential equations of a special kind. The main purpose in [18] was to obtain an intrinsic, geometrical characterization of such systems, and to explain the basic underlying concepts in a brief note. In the present paper we address the more intricate part of the theory. It involves in the first place understanding all details of an algorithmic construction of quadratic integrals and their involutivity. It secondly requires explaining the subtle way in which suitably constructed canonical transformations reduce the HamiltonJacobi problem of the (a priori time-dependent) driven part of the system into that of an equivalent autonomous system of Stäckel type.
\end{abstract}

\section{Introduction}

To the best of our knowledge, the idea of a cofactor system stems from a paper by RauchWojciechowski et al. on certain Newtonian systems in the Euclidean plane [16], but the term 'quadratic integral of cofactor type' came in use with Lundmark's generalization to systems of arbitrary dimension, which was published with some delay in [9]. The term refers to the fact that the matrix of the quadratic part of the first integral comes from the cofactor tensor of a tensor which has special properties with respect to the (Euclidean) metric of the kinetic energy of the system (a tensor which was termed 'inertia tensor' in Benenti's work [1] on Stäckel systems). The other point to be emphasized about the 'quadratic integral of cofactor type' is that the zeroth-order terms in the integral do not come from a potential energy function, in other words, the Newtonian systems under consideration have force terms which are of nonconservative type, albeit of a very special nature, determined by a scalar function also and by the cofactor tensor. It was recognized in [5] that the work of Lundmark could easily be generalized to kinetic energy terms associated to an arbitrary Riemannian metric and in fact fits perfectly within the theory of 'special conformal Killing tensors', as studied in [6]. This gave rise to an intrinsic 
characterization of general cofactor systems, in the context of which it was shown that the corresponding quadratic integral in fact is the Hamiltonian for a quasi-Hamiltonian representation of the system with respect to a non-standard Poisson structure, coming from the special conformal Killing tensor (scKt for short). It must be said that scKts for general metrics had already appeared also, with slightly different assumptions and under different names, in the work of Benenti (see the comprehensive review paper [3]).

As our title reflects, there is an extra aspect about the cofactor systems we want to study. So called 'driven cofactor systems' again were introduced by Lundmark and RauchWojciechowski [10], still in the context of mechanical systems with Euclidean kinetic energy metric and hence having no terms quadratic in the velocities in the second-order equations of motion. Briefly, the systems discussed in [10] are of the form

$$
\begin{aligned}
\ddot{y}^{i} & =Q^{i}\left(y^{j}\right), \quad i=1, \ldots, m \\
\ddot{x}^{a} & =Q^{a}\left(y^{i}, x^{b}\right) \quad a=1, \ldots, n .
\end{aligned}
$$

To begin with, they exhibit a given partial decoupling whereby the $y$-equations are referred to as the driving system, and the $x$-equations as the driven part. In addition, it is assumed that the overall system is of cofactor type and that, more restrictively, the force terms $Q^{a}$ come from a potential, parametrically depending on the driving coordinates. These are rather strong conditions indeed, but they were shown to lead to quite striking conclusions in [10]. First of all the driving system turns out to be of cofactor type in its own right. Secondly, the driven system, when regarded as a time-dependent system along solutions $y(t)$ of the driving system, has $n$ (time-dependent) quadratic integrals. Most astonishingly, however, the authors managed to show that (under some technical assumptions) there exists a time-dependent standard canonical transformation, which has the effect of shifting the time-dependence to an overall factor, so that an autonomous Hamiltonian can be identified which turns out to be of Stäckel type.

Clearly, such results lead to a double challenge. The first question is whether they can be extended to general cofactor systems and can be understood in more intrinsic geometric terms this way. Secondly, if there exists an intrinsic scheme behind these observations, coordinates with respect to which the system partially decouples should not be regarded as part of the data; their existence and a constructive procedure to find them should follow from testing coordinate free conditions. This second aspect fortunately has sufficiently been explored in the literature. Second-order equations with such a decoupling property were called submersive in [8], where both local and global criteria were developed for their characterization. The local conditions for existence of appropriate coordinates and for their construction were turned into a more compact and transparent form by using the geometric calculus along the tangent bundle projection developed in [15] (see also [12]).

In a brief communication [18], one of us has presented the main ingredients towards the resolution of the above double challenge. First of all, [18] provides a coordinate free definition of a driven cofactor system. It further shows that the driving system carries a cofactor structure in its own right and that the overall system has a second, in some sense degenerate cofactor representation, from which one can develop a scheme to construct $n+1$ quadratic integrals. One of these is the integral corresponding to the cofactor nature of 
the driving system; the other $n$ (the dimension of the driven system) are integrals of the driven system along solutions of the driving one. What was not sufficiently understood at that time was the delicate issue of the nature of a canonical transformation (if any) which would have the effect of eliminating the time-dependence form the driven system in a way which leads to the identification of a Stäckel system, as in the Euclidean case. The main purpose of the present paper is precisely to complete this part of the story. To understand precisely what is happening in the process, we need much more explicit information about the recursive procedure which leads to the $n+1$ first integrals referred to before, and also about the precise structure of these integrals. The details of such computations will take a substantial part of our discussion, but in doing so, the present paper will also complement a number of the results already mentioned in [18], where the focus was more on existence issues.

The next section contains the basic definitions needed to describe driven cofactor systems and a summary of results presented in [18]. In Section 3, we develop the algorithm which leads to the identification of the $n+1$ first integrals referred to before. A key issue for understanding the nature of the driven system is the identification of a scKt for its proper metric. It is shown in Section 4 that this scKt does not, however, turn the driven system into a cofactor system in its own right; instead it does that for a modified driven system which turns out to play a role further on. In Section 5, we start by identifying Darboux coordinates for the symplectic form associated to the scKt of the complete system, they are obtained by suitably modifying the momenta. But we gradually develop arguments then to come to an even better selection of modified momenta, which takes the specific decoupling properties of our system into account and are shown to be related to a timedependent (standard) canonical transformation for the driven part of the system. In Section 6, we prove that the application of this canonical transformation, followed by one which comes from using eigenfunctions as new coordinates, produces the rather miraculous effect of reducing the driven system essentially to an autonomous Stäckel type system. The proofs in Section 6 are partly based on simple, indirect arguments, but they are supported also by explicit computations about the structure of all first integrals, which are presented in an Appendix. A couple of illustrative examples are presented in Section 7.

\section{Preliminaries}

The mechanical systems we are talking about in this paper belong to the class of nonconservative Lagrangian systems, governed by equations of the form

$$
\frac{d}{d t}\left(\frac{\partial T}{\partial \dot{q}^{\alpha}}\right)-\frac{\partial T}{\partial q^{\alpha}}=Q_{\alpha} .
$$

Here $T=\frac{1}{2} g_{\alpha \beta}(q) v^{\alpha} v^{\beta}$ is a kinetic energy function on the tangent bundle $T M$ of a Riemannian manifold $M$ with metric $g$; the nonconservative forces $Q_{\alpha}$ are assumed to depend on the position variables only and thus can be viewed as components of a 1-form $\mu=Q_{\alpha}(q) d q^{\alpha}$ on $M$. We recall from [6] that a special conformal Killing tensor $J$ (scKt) 
is a type $(1,1)$ tensor field on $M$, which is symmetric with respect to $g$ and satisfies (lowering an index in the usual way)

$$
J_{\alpha \beta \mid \gamma}=\frac{1}{2}\left(\alpha_{\alpha} g_{\beta \gamma}+\alpha_{\beta} g_{\alpha \gamma}\right), \quad \text { which further implies that } \alpha=d(\operatorname{tr} J) .
$$

The Nijenhuis torsion $\mathcal{N}_{J}$ vanishes, implying that $d_{J}^{2}=0$. Recall for completeness (see [7]) that $d_{J}$ is the derivation of degree 1 which (anti)commutes with the exterior derivative and whose action on functions $f \in C^{\infty}(M)$ is given by $d_{J} f=J(d f)$. The Nijenhuis torsion $\mathcal{N}_{J}$ is the vector-valued 2 -form with components

$$
J_{\beta}^{\alpha}\left(\frac{\partial J_{\gamma}^{\beta}}{\partial q^{\delta}}-\frac{\partial J_{\delta}^{\beta}}{\partial q^{\gamma}}\right)-J_{\delta}^{\beta} \frac{\partial J_{\gamma}^{\alpha}}{\partial q^{\beta}}+J_{\gamma}^{\beta} \frac{\partial J_{\delta}^{\alpha}}{\partial q^{\beta}} .
$$

We also have that $D_{J}^{2}=0$, where $D_{J}$ is the 'gauged differential operator', defined by

$$
D_{J} \rho=d_{J} \rho+d(\operatorname{tr} J) \wedge \rho=(\operatorname{det} J)^{-1} d_{J}((\operatorname{det} J) \rho) .
$$

The equality of both expressions in the defining relation of $D_{J}$ follows from the fact that

$$
d_{J}(\operatorname{det} J)=(\operatorname{det} J) d(\operatorname{tr} J) \text {, }
$$

for any tensor with vanishing Nijenhuis torsion.

The concept of a cofactor system on a general Riemannian manifold was introduced in [5] in the following way.

Definition 1. A cofactor system is a triple $(g, \mu, J)$ on a Riemannian manifold where $g$ is the metric, $\mu$ is a 1-form and $J$ is a nonsingular special conformal Killing tensor such that $D_{J} \mu=0$.

Combining this concept with the geometric notion of submersiveness, we came in [18] to the following generalization and coordinate free formulation of the kind of driven cofactor systems introduced in [10].

Definition 2. A driven cofactor system is a cofactor system $(g, \mu, J)$, for which there exists a distribution $K$ along the projection $\tau: T M \rightarrow M$, with the properties

$$
\begin{gathered}
\Phi(K) \subset K, \quad \nabla K \subset K, \quad \mathrm{D}_{Z}^{V} K \subset K \quad \forall Z \in \mathcal{X}(\tau), \\
d \mu(K, K)=0, \quad \mathrm{D}^{H} \mu\left(K^{\perp}, K\right) \neq 0 .
\end{gathered}
$$

To understand the meaning of these conditions, one needs to know about the following intrinsic geometrical concepts associated to general second-order differential equation fields (SODEs), say

$$
\Gamma=v^{\alpha} \frac{\partial}{\partial q^{\alpha}}+f^{\alpha}(q, v) \frac{\partial}{\partial v^{\alpha}} .
$$

Such a SODE comes with a connection, determined by the following horizontal lift construction from $\mathcal{X}(M)$ (the module of vector fields on $M$ ) to $\mathcal{X}(T M)$

$$
X=X^{\alpha}(q) \frac{\partial}{\partial q^{\alpha}} \mapsto X^{H}=X^{\alpha} H_{\alpha},
$$


where

$$
H_{\alpha}=\frac{\partial}{\partial q^{\alpha}}-\Gamma_{\alpha}^{\beta} \frac{\partial}{\partial v^{\beta}}, \quad \text { with } \quad \Gamma_{\beta}^{\alpha}=-\frac{1}{2} \frac{\partial f^{\alpha}}{\partial v^{\beta}} .
$$

$\Gamma_{\beta}^{\alpha}$ are the connection coefficients. Naturally, we also have a vertical lift

$$
X=X^{\alpha}(q) \frac{\partial}{\partial q^{\alpha}} \mapsto X^{V}=X^{\alpha} V_{\alpha}, \quad \text { where } V_{\alpha}=\frac{\partial}{\partial v^{\alpha}}
$$

It is clear that both operations still make sense if we allow the components of $X$ to be functions $X^{\alpha}(q, v)$, meaning that we extend the domain of the horizontal and vertical lift to $\mathcal{X}(\tau)$, the $C^{\infty}(T M)$-module of vector fields along $\tau: T M \rightarrow M$. These lifts further give rise to corresponding horizontal and vertical covariant derivative operators $\mathrm{D}_{X}^{H}$ and $\mathrm{D}_{X}^{V}$, determined by the following action on functions $F \in C^{\infty}(T M)$ and basic vector fields (and then further extended by duality):

$$
\begin{array}{ll}
\mathrm{D}_{X}^{V} F=X^{\alpha} V_{\alpha}(F), & \mathrm{D}_{X}^{V} \frac{\partial}{\partial q^{\alpha}}=0 \\
\mathrm{D}_{X}^{H} F=X^{\alpha} H_{\alpha}(F), & \mathrm{D}_{X}^{H} \frac{\partial}{\partial q^{\alpha}}=X^{\beta} V_{\alpha}\left(\Gamma_{\beta}^{\gamma}\right) \frac{\partial}{\partial q^{\gamma}} .
\end{array}
$$

The $\mathrm{D}^{H}$ in (6) is a covariant differential, defined for any tensor field $\mathcal{T}$ along $\tau$ by $\mathrm{D}^{H} \mathcal{T}(X, Y, \ldots)=\mathrm{D}_{X}^{H} \mathcal{T}(Y, \ldots)$. Furthermore, the decomposition of $\mathcal{L}_{\Gamma} X^{H}$ into its horizontal and vertical part identifies the important concepts of dynamical covariant derivative $\nabla$, a self-dual degree 0 derivation on tensor fields along $\tau$, and Jacobi endomorphism, a $(1,1)$ tensor $\Phi$ along $\tau$ :

$$
\mathcal{L}_{\Gamma} X^{H}=(\nabla X)^{H}+\Phi(X)^{V} .
$$

For practical purposes, it suffices to know that:

$$
\begin{gathered}
\nabla F=\Gamma(F) \quad \nabla \frac{\partial}{\partial q^{\alpha}}=\Gamma_{\alpha}^{\beta} \frac{\partial}{\partial q^{\beta}} \quad \nabla d q^{\alpha}=-\Gamma_{\beta}^{\alpha} d q^{\beta}, \\
\Phi_{\beta}^{\alpha}=-\frac{\partial f^{\alpha}}{\partial q^{\beta}}-\Gamma_{\gamma}^{\alpha} \Gamma_{\beta}^{\gamma}-\Gamma\left(\Gamma_{\beta}^{\alpha}\right) .
\end{gathered}
$$

For the broader picture of derivations of forms along $\tau$, where the above mentioned concepts play a distinctive role, one can consult [13, 14].

As was discussed in Proposition 1 of [18] and before that already in [12], the existence of a distribution $K$ along $\tau$ having the properties (5) implies that $K$ is actually spanned by a distribution on $M$ which is integrable, and introducing adapted coordinates $\left(y^{i}, x^{a}\right)$ has the effect that the given soDE partially decouples into equations of the form

$$
\begin{aligned}
\ddot{y}^{i} & =f^{i}(y, \dot{y}), \quad i=1, \ldots, m, \\
\ddot{x}^{a} & =f^{a}(x, y, \dot{x}, \dot{y}), \quad a=1, \ldots, n \quad(\text { here } n+m=N=\operatorname{dim} M) .
\end{aligned}
$$

We will keep referring to the decoupled $y$-system as the driving equations and to the remaining $x$-equations as the driven system. But there is more to it in this context, which brings us to the final requirements (6) in definition 2. $K^{\perp}$ of course is the orthogonal 
complement of $K$ with respect to the Riemannian metric $g$. An important property of $g$ is that

$$
\nabla g=0
$$

which follows from the fact that $g$ is the Hessian of the Lagrangian for the conservative part of the system and the knowledge that the nonconservative forces determined by $\mu$ have no effect on the connection coefficients. It was shown in [18] that as a result of (9) $K^{\perp}$ inherits the properties $\nabla K^{\perp} \subset K^{\perp}$ and $\mathrm{D}_{Z}^{V} K^{\perp} \subset K^{\perp}$ from $K$ and that this is enough to conclude that the two complementary distributions are in fact simultaneously integrable. Hence we can choose $x^{a}$ and $y^{i}$ coordinates which are adapted to $K$ and $K^{\perp}$ at the same time, i.e. in such a way that

$$
K=\operatorname{sp}\left\{\frac{\partial}{\partial x^{a}}\right\}, \quad K^{\perp}=\operatorname{sp}\left\{\frac{\partial}{\partial y^{i}}\right\} .
$$

It also follows that the kinetic energy part in the equations of motion (11) decouples completely. In other words, if we put $g_{1}:=\left.g\right|_{K^{\perp}}$ and $g_{2}:=\left.g\right|_{K}$, then in adapted coordinates:

$$
g_{1}=g_{i j}(y) d y^{i} \otimes d y^{j}, \quad g_{2}=g_{a b}(x) d x^{a} \otimes d x^{b},
$$

while $g_{i a}=g_{a i}=0$. Similarly, for the corresponding connection coefficients, we have

$$
\Gamma_{j k}^{i}=\Gamma_{j k}^{i}(y), \quad \Gamma_{b c}^{a}=\Gamma_{b c}^{a}(x)
$$

and all other connection coefficients are zero. If we insist on keeping some partial coupling between a driving and a driven part of the dynamics therefore, this can only come from the nonconservative forces in $\mu$. The second of the conditions (6) exactly guarantees such a coupling. The condition $d \mu(K, K)=0$ on the other hand can easily be seen to model the additional assumption that, in adapted coordinates, the driven part has force terms $Q_{a}$ which are derivable from a potential energy function (with parametric dependence on the driving coordinates $y^{i}$ ).

\section{The cofactor pair scheme on $M$ and $n+1$ quadratic integrals}

It is appropriate to introduce complementary projection operators

$$
P_{1}: \mathcal{X}(M) \rightarrow K^{\perp}, \quad P_{2}: \mathcal{X}(M) \rightarrow K
$$

We thus have $P_{1}+P_{2}=I_{N}$ (the identity tensor on the $N$-dimensional manifold $M$ ), $P_{1} \circ P_{2}=P_{2} \circ P_{1}=0, P_{i}^{2}=P_{i}$, and we occasionally put $\left.P_{1}\right|_{K^{\perp}}=I_{m},\left.P_{2}\right|_{K}=I_{n}$. As in [18], we then look at the scKt tensor $J$ (for its action on vector fields) as the sum of the following four parts

$$
J_{i}=P_{i} \circ J \circ P_{i}, i=1,2, \quad J_{12}=P_{1} \circ J \circ P_{2}, \quad J_{21}=P_{2} \circ J \circ P_{1},
$$


and we shall use a similar notation also for other type $(1,1)$ tensor fields of interest further on.

IMPORTANT NOTATIONAL CONVENTION: In principle, such tensor fields act on the whole module of vector fields on $M$; we shall use the same notation, however, when we consider their restriction to the appropriate submodule $K$ or $K^{\perp}$ where they are not zero.

Recall that the scKt conditions (2), when expressed in terms of the coefficients of the original type $(1,1)$ tensor, take the form

$$
J_{\beta \mid \gamma}^{\alpha}:=\frac{\partial J_{\beta}^{\alpha}}{\partial q^{\gamma}}-J_{\sigma}^{\alpha} \Gamma_{\beta \gamma}^{\sigma}+J_{\beta}^{\sigma} \Gamma_{\sigma \gamma}^{\alpha}=\frac{1}{2}\left(\alpha_{\beta} \delta_{\gamma}^{\alpha}+\alpha_{\sigma} g^{\sigma \alpha} g_{\beta \gamma}\right) .
$$

Taking the decoupling properties of $g$ into account, it follows that in adapted coordinates: $\partial J_{j}^{i} / \partial x^{a}=\partial J_{b}^{a} / \partial y^{i}=0$ and

$$
J_{i \mid k}^{a}=\frac{\partial J_{i}^{a}}{\partial y^{k}}-J_{j}^{a} \Gamma_{i k}^{j}=\frac{1}{2} \alpha_{b} g^{b a} g_{i k}, \quad J_{b \mid a}^{i}=\frac{\partial J_{b}^{i}}{\partial x^{a}}-J_{c}^{i} \Gamma_{b a}^{c}=\frac{1}{2} \alpha_{j} g^{j i} g_{a b} .
$$

Hence, the different blocks of $J$ have the following type of restricted dependence on the adapted coordinates:

$$
J_{1}=J_{j}^{i}(y) \frac{\partial}{\partial y^{i}} \otimes d y^{j}, \quad J_{2}=J_{b}^{a}(x) \frac{\partial}{\partial x^{a}} \otimes d x^{b}
$$

while

$$
J_{21}=J_{i}^{a}(y, x) \frac{\partial}{\partial x^{a}} \otimes d y^{i}, \quad J_{12}=J_{a}^{i}(y, x) \frac{\partial}{\partial y^{i}} \otimes d x^{a}
$$

with

$$
\frac{\partial J_{i}^{a}}{\partial y^{k}}=\frac{\partial J_{k}^{a}}{\partial y^{i}} \quad \text { and } \quad \frac{\partial J_{a}^{i}}{\partial x^{b}}=\frac{\partial J_{b}^{i}}{\partial x^{a}} .
$$

Furthermore, with $\mu_{i}:=P_{i}(\mu)$ and assuming that $J_{1}$ is nonsingular, we know from [18] that $\left(g_{1}, \mu_{1}, J_{1}\right)$ provides a cofactor representation of the driving system. This implies in particular (see further for more details) that this system has a quadratic integral

$$
E^{1}=\frac{1}{2} A_{i j}^{1}(y) \dot{y}^{i} \dot{y}^{j}+W^{1}(y),
$$

where $A^{1}=\operatorname{cof} J_{1}$. For clarity: the cofactor tensor $A$ of a type $(1,1)$ tensor $J$ (notation $A=\operatorname{cof} J)$ is defined by the relation $J A=A J=(\operatorname{det} J) I$. The function $W^{1}$ is determined by the relation $A^{1} \mu_{1}=-d W^{1}$, which is locally equivalent to the condition $D_{J_{1}} \mu_{1}=0$ in the definition of a cofactor system. Incidentally, the minus sign in the expression for $A^{1} \mu_{1}$ is in correspondence with the $+W^{1}$ in the integral $E^{1}$ (and [18] contains a sign error in this sense).

We finally recall from [18] that $P_{2}$, being a degenerate type $(1,1)$ tensor field on $M$, formally satisfies the requirements for a scKt with respect to $g$, and additionally has the property $D_{P_{2}} \mu=0$, meaning that we have a second (degenerate) cofactor representation for the full nonconservative system $\Gamma$, the implications of which we will investigate now. The starting point is that $J+a P_{2}$ also satisfies the scKt condition for any constant $a$ (and 
is nonsingular for sufficiently small values of $a$ ). Let $A(a)$ denote the cofactor tensor of $J+a P_{2}$, so that

$$
\left(J+a P_{2}\right) A(a)=A(a)\left(J+a P_{2}\right)=\operatorname{det}\left(J+a P_{2}\right) I_{N} .
$$

Since $P_{2}=I_{n}$ in adapted coordinates, it is clear that $A(a)$ and $\operatorname{det}\left(J+a P_{2}\right)$ are polynomials in $a$ of degree $n$. We represent them as follows,

$$
A(a)=\sum_{i=1}^{n+1} A_{(i)} a^{i-1}, \quad \operatorname{det}\left(J+a P_{2}\right)=\sum_{i=1}^{n+1} \Delta_{(i)} a^{i-1}
$$

and identify the coefficients of equal powers of $a$ in the identities (16). We get

$$
\begin{aligned}
P_{2} A_{(n+1)} & =A_{(n+1)} P_{2}=0, \\
J A_{(i+1)}+P_{2} A_{(i)} & =A_{(i+1)} J+A_{(i)} P_{2}=\Delta_{(i+1)} I_{N}, \quad(1 \leq i \leq n), \\
J A_{(1)} & =A_{(1)} J=\Delta_{(1)} I_{N} .
\end{aligned}
$$

Information about the block structure of the different $A_{(i)}$ should follow by left and right actions of the projectors $P_{k}$ on these relations. It immediately follows from (17) for example that

$$
A_{(n+1) 21}=A_{(n+1) 12}=A_{(n+1) 2}=0,
$$

while (18) with $i=n$ subsequently implies that $J_{1} A_{(n+1) 1}=A_{(n+1) 1} J_{1}=\Delta_{(n+1)} P_{1}$, or in the restriction to $K^{\perp}$ :

$$
J_{1} A_{(n+1) 1}=A_{(n+1) 1} J_{1}=\Delta_{(n+1)} I_{m} .
$$

Taking into account that terms of degree $n$ in $A(a)$ can only be produced by minors of the $J_{1}$ elements of $J$, this implies that

$$
A_{(n+1) 1}=A^{1}=\operatorname{cof} J_{1} \quad \text { and } \quad \Delta_{(n+1)}=\operatorname{det} J_{1} .
$$

The same equation (18) for $i=n$ then further yields information about parts of $A_{(n)}$ : it is indeed not hard to show by appropriate actions of the projectors that (in the restriction to $K$ or $K^{\perp}$ )

$$
A_{(n) 2}=\left(\operatorname{det} J_{1}\right) I_{n}, \quad A_{(n) 21}=-J_{21}\left(\operatorname{cof} J_{1}\right), \quad A_{(n) 12}=-\left(\operatorname{cof} J_{1}\right) J_{12} .
$$

For the remaining part of $A_{(n)}$, we have to move to the next line in the hierarchy $(i=n-1$ in (18)): a right and left action of $P_{1}$ leads to $J_{1} A_{(n) 1}+J_{12} A_{(n) 21}=\Delta_{(n)} P_{1}$, from which it readily follows that

$$
A_{(n) 1}=\left(\operatorname{det} J_{1}\right) J_{1}^{-1} J_{12} J_{21} J_{1}^{-1}+\Delta_{(n)} J_{1}^{-1}
$$

We will come back in more detail to the continuation of this recursive scheme when we are in a position to gather information about the functions $\Delta_{(i)}$. But note that the final relation (19) expresses that $A_{(1)}=\operatorname{cof} J$ and $\Delta_{(1)}=\operatorname{det} J$.

The important feature about having a cofactor pair scheme is that it gives rise to a family of quadratic integrals which are in involution with respect to a double Poisson 
structure. We sketch how this will work here by following the procedure explained in [5]. Let $\hat{g}: T M \rightarrow T^{*} M$ denote the Legendre type diffeomorphism coming from the given metric $g$, which in (general) coordinates reads $p_{\alpha}=g_{\alpha \beta} v^{\beta}$, and put $\hat{\Gamma}=\hat{g}_{*} \Gamma$, where $\Gamma$ is the SODE defined by the cofactor system $(g, \mu, J)$. Then, according to theorem 2 in [5], the requirements about a cofactor system are equivalent to $\hat{\Gamma}$ representing a quasi-Hamiltonian system with a quadratic Hamiltonian with respect to the Poisson tensor which can be constructed from the tensor $J$ with vanishing Nijenhuis torsion. More precisely, if $\tilde{J}$ denotes the so-called complete lift of $J$ to $T^{*} M$ and $P_{0}$ is the canonical Poisson map on $T^{*} M$, i.e. the canonical Poisson tensor, interpreted as a map $P_{0}: \mathcal{X}^{*}\left(T^{*} M\right) \rightarrow \mathcal{X}\left(T^{*} M\right)$, we put $P_{J}=\tilde{J} \circ P_{0}$ and the point about $\hat{\Gamma}$ is that

$$
(\operatorname{det} J) \hat{\Gamma}=P_{J}(d H), \quad \text { with } \quad H=\frac{1}{2} A^{\alpha \beta} p_{\alpha} p_{\beta}+W,
$$

where $A$ is the cofactor tensor of $J$ and $W$ is the function, determined (up to a constant) by the property $A \mu=-d W$. Having a double cofactor representation with special conformal Killing tensors such as $J$ and $P_{2}$ in our present situation, will entail that $\hat{\Gamma}$ satisfies a relation of the form

$$
\left(\operatorname{det}\left(J+a P_{2}\right)\right) \hat{\Gamma}=P_{J+a P_{2}} d H(a), \quad \text { with } \quad H(a)=\frac{1}{2} A^{\alpha \beta}(a) p_{\alpha} p_{\beta}+W(a) .
$$

Since $P_{J+a P_{2}}=P_{J}+a P_{P_{2}}$, one can see that $H(a)$ will be a polynomial of degree at most $n$ in the parameter $a$, say of the form

$$
H(a)=\sum_{i=1}^{n+1} H_{(i)} a^{i-1}, \quad \text { with } \quad H_{(i)}=\frac{1}{2} A_{(i)}^{\alpha \beta} p_{\alpha} p_{\beta}+W_{(i)},
$$

where $A_{(i)}^{\alpha \beta}$ comes from the $A_{(i)}$ tensor considered before, with an index raised by the given metric $g$, and $W_{(i)}$ is determined by the relation $A_{(i)} \mu=-d W_{(i)}$. Naturally $H(a)$ and therefore all its coefficients $H_{(i)}$ will be first integrals of the system $\hat{\Gamma}$.

Before proceeding, observe that $H_{(n+1)}$ is essentially the function $E^{1}$ which is a first integral of the driving system (more precisely $H_{(n+1)}=g_{*} E^{1}$ ). Indeed, it follows from the block structure of $A_{(n+1)}$ determined before that in adapted coordinates, with $p_{i}=$ $g_{i j}(y) \dot{y}^{j}$,

$$
H_{(n+1)}=\frac{1}{2}\left(A^{1}\right)^{i j}(y) p_{i} p_{j}+W^{1}(y) .
$$

It is worth mentioning also that for a general $J$, the coordinate expression of $P_{J}$ is given by

$$
P_{J}=J_{\beta}^{\alpha} \frac{\partial}{\partial p_{\beta}} \wedge \frac{\partial}{\partial q^{\alpha}}-\frac{1}{2} p_{\gamma}\left(\frac{\partial J_{\alpha}^{\gamma}}{\partial q^{\beta}}-\frac{\partial J_{\beta}^{\gamma}}{\partial q^{\alpha}}\right) \frac{\partial}{\partial p_{\alpha}} \wedge \frac{\partial}{\partial p_{\beta}} .
$$

Naturally, in adapted coordinates,

$$
P_{P_{2}}=\frac{\partial}{\partial p_{a}} \wedge \frac{\partial}{\partial x^{a}}
$$

where the momentum variables $p_{a}$ are defined by $p_{a}=g_{a b}(x) \dot{x}^{b}$. 
Taking the various polynomial representations into account, property (25) becomes

$$
\left(\sum_{i=1}^{n+1} \Delta_{(i)} a^{i-1}\right) \hat{\Gamma}=\left(P_{J}+a P_{P_{2}}\right)\left(\sum_{i=1}^{n+1} d H_{(i)} a^{i-1}\right) .
$$

Identifying coefficients of equal powers of a requires first of all that we should have $P_{P_{2}}\left(d H_{(n+1)}\right)=0$, and this is clearly verified in view of the preceding observations. We further must have that

$$
\Delta_{(i)} \hat{\Gamma}=P_{J}\left(d H_{(i)}\right)+P_{P_{2}}\left(d H_{(i-1)}\right), \quad 1<i \leq n+1
$$

and finally for $i=1$ that $\Delta_{(1)} \hat{\Gamma}=P_{J}\left(d H_{(1)}\right)$, but this is merely a confirmation of the quasi-Hamiltonian structure coming from $J$, since $\Delta_{(1)}=\operatorname{det} J$ and $H_{(1)}=H$. There is

no reason to expect that the $n$ first integrals $H_{(1)}$ up to $H_{(n)}$ of $\hat{\Gamma}$ would depend on the coordinates $\left(x^{a}, p_{a}\right)$ only. Nevertheless, they will be first integrals of the driven system along solutions $\left(y^{i}(t), p_{i}(t)\right)$ of the driving system. As for the question of involutiveness, if we adopt the notational convention that $P_{J}(d f)=\{f, \cdot\}_{J}$, it follows from (30) that

$$
\left\{H_{(i)}, H_{(l)}\right\}_{J}+\left\{H_{(i-1)}, H_{(l)}\right\}_{P_{2}}=\Delta_{(i)} \hat{\Gamma}\left(H_{(l)}\right)=0, \quad 1<i \leq n+1,1 \leq l \leq n+1,
$$

and from the two other observations about $H_{(n+1)}$ and $H_{(1)}$ that

$$
\left\{H_{(1)}, H_{(l)}\right\}_{J}=\left\{H_{(n+1)}, H_{(l)}\right\}_{P_{2}}=0 \quad \forall l .
$$

Using (32), it further follows from (31) with $l=1$ and $l=n+1$ respectively, that also

$$
\left\{H_{(l)}, H_{(1)}\right\}_{P_{2}}=\left\{H_{(l)}, H_{(n+1)}\right\}_{J}=0,
$$

and then a simple recursive argument in (31) finally implies that all $H_{(i)}$ are in involution with respect to both the $J$-bracket and the $P_{2}$-bracket. It is worth observing (see (29)) that in adapted coordinates the $P_{2}$-bracket formally looks like the standard Poisson bracket in the $\left(x^{a}, p_{a}\right)$ coordinates so that we have, along solutions of the driving system, $n$ integrals for the driven system which are in involution in the standard sense.

\section{An induced special conformal Killing tensor for the metric of the driven system}

Part of our basic assumptions so far is that both $J$ and $J_{1}$ are nonsingular. We shall see now that this naturally leads to the introduction of another nonsingular type $(1,1)$ tensor $\bar{J}_{2}$, which is a kind of deformation of $J_{2}$. When talking about nonsingularity here, don't forget the notational convention specified before! To say that $J_{1}$ is nonsingular of course only makes sense when we mean $\left.J_{1}\right|_{K^{\perp}}$; likewise, nonsingularity of $\bar{J}_{2}$ will refer to $\left.\bar{J}_{2}\right|_{K}$.

For later reference we look at the adjoint action of $J$ on 1 -forms for a moment. In doing so (as we already tacitly did for the cofactor tensor $A$ ) we use the same notation again, i.e. do not write $J^{*}$ as is sometimes customary. One has to keep in mind, however, that when compositions are involved (as in the definition of $J_{12}$ and $J_{21}$ ), the order of 
such compositions has to be reversed. Let $\alpha$ be an arbitrary 1-form on $M$ and put $\beta=J \alpha=J\left(P_{1} \alpha+P_{2} \alpha\right)$. To solve such a relation for $\alpha$ in terms of $\beta$, it is natural to actually compute $P_{1} \alpha$ and $P_{2} \alpha$. We have

$$
\begin{aligned}
& P_{1} \beta=J_{1}\left(P_{1} \alpha\right)+J_{21}\left(P_{2} \alpha\right), \\
& P_{2} \beta=J_{12}\left(P_{1} \alpha\right)+J_{2}\left(P_{2} \alpha\right) .
\end{aligned}
$$

Since $J_{1}$ is nonsingular, it follows from the first relation that

$$
P_{1} \alpha=J_{1}^{-1}\left(P_{1} \beta\right)-J_{1}^{-1} J_{21}\left(P_{2} \alpha\right),
$$

and substitution of this result in the second relation leads to

$$
\bar{J}_{2}\left(P_{2} \alpha\right)=\left(P_{2}-J_{12} J_{1}^{-1} P_{1}\right) \beta,
$$

where $\bar{J}_{2}$ is defined (for its action on 1 -forms) as

$$
\bar{J}_{2}=J_{2}-J_{12} J_{1}^{-1} J_{21}
$$

Obviously $\bar{J}_{2}$ vanishes on $K^{\perp}$, but will be nonsingular on $K$ so that $P_{2} \alpha$ now can be obtained from (34) and substitution in (33) subsequently gives us $P_{1} \alpha$. In fact, what we are looking at here is the following typical factorization of a matrix with a block structure, this time written as representing the action of a type $(1,1)$ tensor on vector fields:

$$
\left(\begin{array}{cc}
J_{1} & J_{12} \\
J_{21} & J_{2}
\end{array}\right)=\left(\begin{array}{cc}
J_{1} & 0 \\
J_{21} & 1
\end{array}\right)\left(\begin{array}{cc}
1 & J_{1}^{-1} J_{12} \\
0 & J_{2}-J_{21} J_{1}^{-1} J_{12}
\end{array}\right) .
$$

It follows that $\operatorname{det} J=\left(\operatorname{det} J_{1}\right)\left(\operatorname{det} \bar{J}_{2}\right)$ so that nonsingularity of $J$ and $J_{1}$ implies the same for $\bar{J}_{2}$. In adapted coordinates, $\bar{J}_{2}$ has components

$$
\bar{J}_{2 b}^{a}=J_{b}^{a}-J_{i}^{a}\left(J_{1}^{-1}\right)_{j}^{i} J_{b}^{j} .
$$

Proposition 1. $\bar{J}_{2}$ is a (parameter dependent) special conformal Killing tensor for $g_{2}$, and its cofactor tensor is $\left(\operatorname{det} J_{1}\right)^{-1} A_{2}$ with $A=\operatorname{cof} J$.

ProOF. The proof is a straightforward computation, for which it will be suitable to work in the adapted $\left(y^{i}, x^{a}\right)$ coordinates. Let us lower an index to apply the scKt condition in the covariant form (2). Keeping in mind that $g_{a i}=g_{i a}=0$, we have

$$
\bar{J}_{2 c b}:=g_{c a} \bar{J}_{2 b}^{a}=J_{c b}-J_{c i}\left(J_{1}^{-1}\right)^{i j} J_{j b} .
$$

Since $J$ is symmetric, the same is true for $\bar{J}_{2}$. Note that $\bar{J}_{2}$ depends on both sets of coordinates, but the $y^{i}$ are regarded as external parameters for our present considerations. Since $J_{1}$ depends on the $y^{i}$ only and the same of course holds for its inverse (or its cofactor tensor $\left.A_{(n+1) 1}\right)$, we get in the first place that

$$
\bar{J}_{2 c b \mid a}=J_{c b \mid a}-J_{c i \mid a}\left(J_{1}^{-1}\right)^{i j} J_{j b}-J_{c i}\left(J_{1}^{-1}\right)^{i j} J_{j b \mid a} .
$$


It follows from (2) that

$$
J_{c b \mid a}=\frac{1}{2}\left(\alpha_{c} g_{b a}+\alpha_{b} g_{c a}\right), \quad \text { and } \quad J_{c i \mid a}=\frac{1}{2} \alpha_{i} g_{c a} .
$$

We then easily obtain that

$$
\bar{J}_{2 c b \mid a}=\frac{1}{2}\left(\bar{\alpha}_{c} g_{b a}+\bar{\alpha}_{b} g_{c a}\right), \quad \text { with } \quad \bar{\alpha}_{c}=\alpha_{c}-J_{c i}\left(J_{1}^{-1}\right)^{i j} \alpha_{j},
$$

which shows that $\bar{J}_{2}$ is a scKt for $g_{2}$.

If we denote cof $J$ as in (24) by $A$ (and we have seen that it is also $A_{(1)}$ ), we know that $(\operatorname{det} J) g^{a c}=J_{\beta}^{a} A^{\beta c}=J_{i}^{a} A^{i c}+J_{b}^{a} A^{b c}$ and $0=(\operatorname{det} J) g^{j c}=J_{k}^{j} A^{k c}+J_{b}^{j} A^{b c}$. It then follows that

$$
\begin{aligned}
\bar{J}_{2 b}^{a} A^{b c}\left(\operatorname{det} J_{1}\right)^{-1} & =\left(\operatorname{det} J_{1}\right)^{-1}\left(J_{b}^{a} A^{b c}-J_{i}^{a}\left(J_{1}^{-1}\right)_{j}^{i} J_{b}^{j} A^{b c}\right) \\
& =\left(\operatorname{det} J_{1}\right)^{-1}\left((\operatorname{det} J) g^{a c}-J_{i}^{a} A^{i c}+J_{i}^{a}\left(J_{1}^{-1}\right)_{j}^{i} J_{k}^{j} A^{k c}\right) \\
& =\left(\operatorname{det} J_{1}\right)^{-1}(\operatorname{det} J) g^{a c} \\
& =\left(\operatorname{det} \bar{J}_{2}\right) g^{a c}
\end{aligned}
$$

which proves the last statement of the proposition.

Note that $\bar{J}_{2}$, perhaps rather unexpectedly, does not give rise to a cofactor system representation of the driven system in a strict sense. In other words, it does not seem to be true that the forces $\mu_{2}$ of the driven system have the property $D_{\bar{J}_{2}} \mu_{2}=0$, or equivalently that (cof $\left.\bar{J}_{2}\right) \mu_{2}$ is closed. At this moment, the closest we can get to such a property is that an associated driven cofactor system $\left(g_{2}, \bar{\mu}_{2}, \bar{J}_{2}\right)$ exists, with modified nonconservative forces $\bar{\mu}_{2}$.

Proposition 2. A cofactor system, parametrically depending on the coordinates of the driving system, is determined by the triple $\left(g_{2}, \bar{\mu}_{2}, \bar{J}_{2}\right)$, where $g_{2}$ and $\bar{J}_{2}$ are as before, and

$$
\bar{\mu}_{2}=P_{2}\left(d W_{(n)}\right),
$$

$W_{(n)}$ being the function encountered in the recursive scheme following from (25).

ProOF. We know that the given nonconservative forces $\mu$ satisfy the relation $A \mu=-d W$, or equivalently $(\operatorname{det} J) \mu=-J(d W)$. Consider the scheme of the beginning of this section which led to the introduction of $\bar{J}_{2}$. Letting $-d W$ play the role of $\alpha$ and $(\operatorname{det} J) \mu$ the role of $\beta$, we put $P_{i}(d W)=d_{i} W$ for convenience. The relation (34) becomes

$$
-\bar{J}_{2}\left(d_{2} W\right)=(\operatorname{det} J) \mu_{2}+\left(\operatorname{det} \bar{J}_{2}\right) J_{12}\left(d_{1} W^{1}\right),
$$

where for the second term on the right we have taken into account that $\mu_{1}$ satisfies $\left(\operatorname{det} J_{1}\right) \mu_{1}=-J_{1}\left(d_{1} W^{1}\right)$ in view of the cofactor representation of the driving system, and that $\operatorname{det} J=\left(\operatorname{det} J_{1}\right)\left(\operatorname{det} \bar{J}_{2}\right)$. Secondly, projecting the relation $A_{(n)} \mu=-d W_{(n)}$ under $P_{2}$, we get in the first place that

$$
A_{(n) 2} \mu_{2}+A_{(n) 12} \mu_{1}=-d_{2} W_{(n)}
$$


Using the information gathered about $A_{(n)}$ in $(22)$ and the cofactor system property of the driving system which was just recalled, this immediately leads to

$$
\left(\operatorname{det} J_{1}\right) \mu_{2}+J_{12}\left(d_{1} W^{1}\right)=-d_{2} W_{(n)} .
$$

Substituting this result in (41), we obtain the relation

$$
\left(\operatorname{det} \bar{J}_{2}\right) d_{2} W_{(n)}=\bar{J}_{2}\left(d_{2} W\right),
$$

which implies, with $\bar{\mu}_{2}=d_{2} W_{(n)}$, that

$$
\left(\operatorname{cof} \bar{J}_{2}\right) \bar{\mu}_{2}=d_{2} W
$$

Together with the knowledge that $\bar{J}_{2}$ is a scKt with respect to $g_{2}$, this expresses that the triple $\left(g_{2}, \bar{\mu}_{2}, \bar{J}_{2}\right)$ determines a cofactor system.

It is worth emphasizing again that the cofactor representation of this modified driven system must be seen also as a statement about second-order differential equations for the $x^{a}$, which parametrically depend on the $y$-coordinates. This is clear, for example, from the fact that the exterior derivative in the picture is $d_{2}$.

As explained in the introduction, one of our main objectives is to explore the remarkable situation that the driven system, although being essentially time-dependent along solutions of the driving system, does give rise in the end to a Stäckel-type Hamilton-Jacobi separability anyway. This will require a supplementary assumption about existence of independent eigenfunctions of $J$. We want to show at this point that it is again the tensor $\bar{J}_{2}$ which is relevant for this purpose.

Consider the (degenerate kind of) eigenvalue equation $\operatorname{det}\left(J-\lambda P_{2}\right)=0$, which is a polynomial equation of degree $n$ for $\lambda$. Our basic assumption now is that this equation has $n$ functionally independent solutions $u^{a}$, in the sense that the 1-forms $P_{2}\left(d u^{a}\right)$ are linearly independent. If we think of the $u^{a}$ as expressed in terms of the adapted $\left(y^{i}, x^{b}\right)$ coordinates, this amounts to saying that the Jacobian $\left(\partial u^{a} / \partial x^{b}\right)$ is nonsingular. From the identity $\operatorname{det}\left(J-u^{a}(y, x) P_{2}\right) \equiv 0$ for each fixed $u^{a}(y, x)$, it follows that

$$
\begin{aligned}
0 & \equiv d\left(\operatorname{det}\left(J-u^{a}(y, x) P_{2}\right)\right) \\
& =\left.d\left(\operatorname{det}\left(J-\lambda P_{2}\right)\right)\right|_{\lambda=u^{a}(y, x)}+\left.\frac{\partial\left(\operatorname{det}\left(J-\lambda P_{2}\right)\right)}{\partial \lambda}\right|_{\lambda=u^{a}(y, x)} d u^{a} .
\end{aligned}
$$

But since $J-\lambda P_{2}$ has vanishing Nijenhuis torsion, we know from (4) that

$$
\left(J-\lambda P_{2}\right) d\left(\operatorname{det}\left(J-\lambda P_{2}\right)\right)=\operatorname{det}\left(J-\lambda P_{2}\right) d \operatorname{tr}\left(J-\lambda P_{2}\right)
$$

for all $\lambda$. It then follows by acting with $\left(J-u^{a}(y, x) P_{2}\right)$ on the preceding identity that

$$
\left.\frac{\partial\left(\operatorname{det}\left(J-\lambda P_{2}\right)\right)}{\partial \lambda}\right|_{\lambda=u^{a}(y, x)}\left(J-u^{a}(y, x) P_{2}\right) d u^{a}=0,
$$

and thus, since all eigenfunctions are assumed to be simple, that

$$
\left(J-u^{a}(y, x) P_{2}\right) d u^{a}=0 .
$$


Proposition 3. Assume that the equation $\operatorname{det}\left(J-\lambda P_{2}\right)=0$ has $n$ functionally independent eigenfunctions $u^{a}$. Then $d u^{a}$ is an eigenform of $J$ (in the sense of equation (45)) corresponding to the eigenvalue $u^{a}$. Moreover, the $u^{a}$ are also eigenfunctions of $\bar{J}_{2}$, with $P_{2}\left(d u^{a}\right)$ as corresponding eigenform.

ProOF. It remains to prove the statement about $\bar{J}_{2}$. For that, it suffices to go back once more to the analysis about $\beta=J \alpha$ at the beginning of this section, with $d u^{a}$ in the role of $\alpha$ and $u^{a} P_{2}\left(d u^{a}\right)$ (no sum!) in the role of $\beta$. The relation (34) then says that

$$
\bar{J}_{2}\left(P_{2}\left(d u^{a}\right)\right)=u^{a} P_{2}\left(d u^{a}\right)
$$

which is precisely what we need.

\section{A symplectic view-point and Darboux coordinates}

Since $J$ is assumed to be nonsingular, the Poisson tensor associated to $P_{J}$ actually comes from a symplectic form which we call $\omega_{J}$. The sign convention which we adopt here is that for any function $F$

$$
X=P_{J}(d F) \quad \Longleftrightarrow \quad i_{X} \omega_{J}=-d F \text {. }
$$

One easily verifies that, referring to the general coordinate expression (28) of $P_{J}, \omega_{J}$ is given by

$$
\omega_{J}=J_{\beta}^{-1}{ }_{\beta}^{\alpha} d p_{\alpha} \wedge d q^{\beta}-\frac{1}{2} p_{\gamma}\left(\frac{\partial J_{\alpha}^{\gamma}}{\partial q^{\beta}}-\frac{\partial J_{\beta}^{\gamma}}{\partial q^{\alpha}}\right){ }_{\sigma}^{-1}{ }_{\sigma}{ }_{\rho}^{-1} d q^{\sigma} \wedge d q^{\rho} .
$$

So far, this correspondence is valid for any nonsingular type $(1,1)$ tensor $J$ on $M$. The first term in $\omega_{J}$ strongly suggests introducing new momentum variables $\check{p}_{\alpha}$ by

$$
p_{\alpha}=J_{\alpha}^{\beta}(q) \check{p}_{\beta}
$$

Lemma 1. The coordinate change $(q, p) \leftrightarrow(q, \check{p})$ determines a Darboux chart for $\omega_{J}$ if and only if $\mathcal{N}_{J}=0$.

Proof. From $\check{p}_{\beta}=J_{\beta}^{-1}{ }_{\beta}^{\alpha} p_{\alpha}$, it is easy to compute that

$$
d \check{p}_{\beta} \wedge d q^{\beta}=J_{\beta}^{-1}{ }_{\beta}^{\alpha} d p_{\alpha} \wedge d q^{\beta}-\frac{1}{2} \check{p}_{\delta}\left(\frac{\partial J_{\tau}^{\delta}}{\partial q^{\sigma}} J_{\rho}^{-1 \tau}-\frac{\partial J_{\tau}^{\delta}}{\partial q^{\rho}} J_{\sigma}^{-1 \tau}\right) d q^{\sigma} \wedge d q^{\rho} .
$$

Subtracting this from (46), one easily obtains that the coefficient of the resulting 2 -form (after multiplication by two $J$-factors) will be zero if and only if $\mathcal{N}_{J}=0$.

As explained in Section 3, the fact that the SODE $\Gamma$ on $T M$ satisfies the requirements of a cofactor system is equivalent to saying that its image $\hat{\Gamma}$ under the Legendre map $\hat{g}$ has a quasi-Hamiltonian representation (24) with respect to $P_{J}$. This in turn translates within the symplectic view-point to $(\operatorname{det} J) i_{\hat{\Gamma}} \omega_{J}=-d H$. The preceding lemma then says that $\omega_{J}$ will take the form of the standard symplectic form on $T^{*} M$ when expressed in the 
variables $(q, \check{p})$. However, it is not clear that we can take advantage of such a coordinate change because it does not take account of the special feature of partial decoupling which our system exhibits. We shall show that there is a better choice of new momenta, which is inspired by the above transition to Darboux coordinates but does take the extra features of a driven cofactor system into account. Note in passing that there exists a different technique for obtaining a Hamiltonian representation out of a quasi-Hamiltonian one: roughly it consists of absorbing the overall factor by a change of timescale, which has the disadvantage, however, that the definition of the new time makes sense only along (the unknown) solutions of the system. This technique is well documented in [3], for example, and was extensively used in the context of cofactor systems in [11. We believe that the line of approach we adopt here offers more insight in understanding the delicate aspects of the driven nature of our cofactor system.

Going back to the SODE $\Gamma$ on $T M$, we can first pass to the coordinates $\left(y^{i}, x^{a}\right)$ adapted to the complementary distributions $K^{\perp}$ and $K$, before passing to the quasi-Hamiltonian representation $\hat{\Gamma}$. The projectors $P_{1}$ and $P_{2}$ have corresponding actions on $T M$ through their complete lifts; they give rise to a partial splitting of $\Gamma$ in the form $\Gamma=\Gamma_{1}+\Gamma_{2}$ say, as exhibited in the equations (7, 8) . Likewise, the complete lifts $\tilde{P}_{1}, \tilde{P}_{2}$ of the projectors to $T^{*} M$ produce a partial decoupling $\hat{\Gamma}=\hat{\Gamma}_{1}+\hat{\Gamma}_{2}$, which in adapted coordinates is simply the effect of transforming (7, 8) to equivalent first-order equations by passing to the momenta $p_{i}=g_{i j}(y) \dot{y}^{j}$ and $p_{a}=g_{a b}(x) \dot{x}^{b}$. It is worth illustrating this in more detail as follows. The SODE $\Gamma$ associated to the equations of motion of the form (1), after applying the overall Legendre map $\hat{g}$, transforms to

$$
\hat{\Gamma}=g^{\alpha \beta} p_{\beta} \frac{\partial}{\partial q^{\alpha}}+\Gamma_{\mu \alpha}^{\gamma} g^{\mu \delta} p_{\gamma} p_{\delta} \frac{\partial}{\partial p_{\alpha}}+Q_{\alpha} \frac{\partial}{\partial p_{\alpha}} .
$$

In adapted $(y, x)$-coordinates, in view of the way the components of $g$ and the connection coefficients decouple (see (10) and its consequences), this expression becomes

$$
\begin{aligned}
\hat{\Gamma}= & g^{i j}(y) p_{j} \frac{\partial}{\partial y^{i}}+\Gamma_{i j}^{k}(y) g^{i l}(y) p_{k} p_{l} \frac{\partial}{\partial p_{j}}+Q_{j}(y) \frac{\partial}{\partial p_{j}} \\
& +g^{a b}(x) p_{b} \frac{\partial}{\partial x^{a}}+\Gamma_{a b}^{c}(x) g^{a d}(x) p_{c} p_{d} \frac{\partial}{\partial p_{b}}+Q_{b}(x, y) \frac{\partial}{\partial p_{b}} .
\end{aligned}
$$

The first line reflects the fact that the driving system has its own cofactor representation, i.e. satisfies

$$
\left(\operatorname{det} J_{1}\right) \hat{\Gamma}_{1}=P_{J_{1}}\left(d H_{(n+1)}\right),
$$

with $H_{(n+1)}$ as in (27). Concerning the second line, we should take into account the extra assumption that the driven system has a standard Hamiltonian representation: as indicated before, the condition $d \mu(K, K)=0$ in definition 2 expresses that the force terms $Q_{a}$ of the driven system are derivable from a potential energy function $V(x, y)$ say, depending parametrically on the driving coordinates $y^{i}$. It is then clear that the second line simply expresses that

$$
\hat{\Gamma}_{2}=P_{P_{2}}(d h), \quad \text { with } \quad h=\frac{1}{2} g^{a b}(x) p_{a} p_{b}+V(x, y),
$$


keeping in mind that $P_{P_{2}}$ in adapted coordinates merely is the standard Poisson structure in the variables $\left(x^{a}, p_{a}\right)$. With this splitting of $\hat{\Gamma}$ in mind, it looks more appropriate not to spoil the decoupled feature of the driving system by introducing Darboux coordinates for the overall symplectic structure $\omega_{J}$. Instead, we can put $p_{i}=J_{1}{ }_{i} \tilde{p}_{j}$, which will have the effect of introducing Darboux coordinates for $\omega_{J_{1}}$. As for the driven part $\hat{\Gamma}_{2}$, let us first investigate in detail what the introduction of the momenta $\check{p}$ would do.

Formally we can regard the transformation formulas $p_{\alpha}=J_{\alpha}^{\beta}(q) \check{p}_{\beta}$ as representing a relation between 1-forms on $M$, of the type $\beta=J \alpha$ discussed at the beginning of Section 4 . It then follows from the considerations leading to (34) that

$$
\bar{J}_{2}^{b} \check{p}_{b}=p_{a}-J_{a}^{i} J_{1}^{-1 j}{ }_{i} p_{j}
$$

This suggests that the more relevant momentum variables for the driven system actually are $\tilde{p}_{a}:=\bar{J}_{2}{ }_{a}^{b} \check{p}_{b}$. The conclusion from this preliminary analysis is that we shall consider the following linear change of momenta

$$
\begin{aligned}
& p_{i}=J_{1}^{j} \tilde{p}_{j}, \\
& p_{a}=\tilde{p}_{a}+J_{a}^{i} \tilde{p}_{i} .
\end{aligned}
$$

It turns out that the transition from $p_{a}$ to $\tilde{p}_{a}$, viewed as time-dependent transformation along solutions of the driving system, actually represents a time-dependent canonical transformation for the driven system in the standard sense and hence is ideally suited to preserve the special assumption on that system. Indeed, in view of the second of the properties (14), we know that the components $J_{a}^{i}$ of $J$ can be written as $J_{a}^{i}=\partial \psi^{i} / \partial x^{a}$ for some functions $\psi^{i}(x, y)$. Defining $F(x, \tilde{p}, t)$ by

$$
F(x, \tilde{p}, t)=x^{a} \tilde{p}_{a}+\Psi(x, t), \quad \text { with } \quad \Psi(x, t)=\psi^{i}(x, y(t)) \tilde{p}_{i}(t),
$$

we create a generating function of mixed type (depending on the old position variables $x$ and new momenta $\tilde{p})$ for a standard canonical transformation $\left(x^{a}, p_{a}\right) \leftrightarrow\left(x^{a}, \tilde{p}_{a}\right)$, which does not change the coordinates, transforms the momenta according to (53), but must be viewed as time-dependent along solutions of the driving system. The Hamiltonian of the transformed system then is given by

$$
\tilde{h}(x, \tilde{p}, t):=h+\frac{\partial F}{\partial t} .
$$

We shall see in the next section that this canonical transformation is one of two steps which are required to relate the original Hamiltonian $h$ of the driven system to the first integral $H_{(n)}$ of $\hat{\Gamma}$ and that $H_{(n)}$ is the key to understanding the subtle way in which the driven system in the end corresponds to an autonomous Hamiltonian system which is separable in the Hamilton-Jacobi sense. 


\section{Separability of the Hamilton-Jacobi equation for the driven system}

Let us start by computing the function $H_{(n)}$ expressed in the variables $\left(y^{i}, x^{a}, \tilde{p}_{i}, \tilde{p}_{a}\right)$. We have

$$
H_{(n)}=\frac{1}{2} A_{(n)}^{a b} p_{a} p_{b}+A_{(n)}^{a i} p_{a} p_{i}+\frac{1}{2} A_{(n)}^{i j} p_{i} p_{j}+W_{(n)} .
$$

From (22), raising an index, we learn that

$$
A_{(n)}^{a b}=\left(\operatorname{det} J_{1}\right) g^{a b}, \quad A_{(n)}^{a i}=-A^{1^{i}}{ }_{l}^{l a} .
$$

Making the substitutions $(52,53)$ it then readily follows (remember that $A^{1}$ is cof $J_{1}$ ) that

$$
H_{(n)}=\frac{1}{2}\left(\operatorname{det} J_{1}\right) g^{a b} \tilde{p}_{a} \tilde{p}_{b}-\frac{1}{2}\left(\operatorname{det} J_{1}\right) J^{b i} J_{b}^{j} \tilde{p}_{i} \tilde{p}_{j}+\frac{1}{2} A_{(n)}^{k l} J_{1 k}^{i} J_{1 l}^{j} \tilde{p}_{i} \tilde{p}_{j}+W_{(n)} .
$$

Using (23) now, we arrive at the following result:

$$
H_{(n)}=\frac{1}{2}\left(\operatorname{det} J_{1}\right) g^{a b} \tilde{p}_{a} \tilde{p}_{b}+\frac{1}{2} \Delta_{(n)} J^{i j} \tilde{p}_{i} \tilde{p}_{j}+W_{(n)} .
$$

So, introducing the new variables has the interesting effect of eliminating the terms in mixed momenta in $H_{(n)}$. Obviously, however, the effect on $h$ will be the opposite. We get

$$
h=\frac{1}{2} g^{a b} \tilde{p}_{a} \tilde{p}_{b}+J^{a i} \tilde{p}_{a} \tilde{p}_{i}+\frac{1}{2} J^{a i} J_{a}^{j} \tilde{p}_{i} \tilde{p}_{j}+V .
$$

But for the interpretation as time-dependent canonical transformation, we need to look at the function $\tilde{h}$ and will show now that this function is more closely related to $H_{(n)}$.

Lemma 2. Under the canonical transformation with generating function (54), the transformed Hamiltonian $\tilde{h}$ of the driven system takes the following form, to within an additive function of time,

$$
\tilde{h}=\left(\operatorname{det} J_{1}\right)^{-1} H_{(n)}+J^{a i} \tilde{p}_{a} \tilde{p}_{i}
$$

Proof. We need to add to the expression (157) for $h$ the term

$$
\frac{\partial F}{\partial t}=\frac{\partial \Psi}{\partial t}=\frac{\partial \Psi}{\partial y^{k}} \dot{y}^{k}+\psi^{k} \dot{\tilde{p}}_{k}
$$

computed along solutions of the driving equations. Since the $\tilde{p}_{i}$ were introduced to provide Darboux coordinates, the Poisson map $P_{J_{1}}$ in (49) takes the form of the standard Poisson map so that the equations of the driving system become

$$
\left(\operatorname{det} J_{1}\right) \dot{y}^{k}=\frac{\partial H_{(n+1)}}{\partial \tilde{p}_{k}}, \quad\left(\operatorname{det} J_{1}\right) \dot{\tilde{p}}_{k}=-\frac{\partial H_{(n+1)}}{\partial y^{k}},
$$

whereby the function $H_{(n+1)}$ from (27), when expressed in the new momenta, reads

$$
H_{(n+1)}=\frac{1}{2}\left(\operatorname{det} J_{1}\right) J_{1}^{i j} \tilde{p}_{i} \tilde{p}_{j}+W^{1} .
$$


It is now fairly straightforward to compute that $\tilde{h}$ can be written as,

$$
\begin{aligned}
\tilde{h}=h & +\frac{\partial \psi^{i}}{\partial y^{k}} J_{1}^{k j} \tilde{p}_{i} \tilde{p}_{j}+J_{1}^{j l} \Gamma_{k l}^{i} \psi^{k} \tilde{p}_{i} \tilde{p}_{j}-\frac{1}{2} J_{1 \mid k}^{i j} \psi^{k} \tilde{p}_{i} \tilde{p}_{j} \\
& -\frac{1}{2}\left(\operatorname{det} J_{1}\right)^{-1} \frac{\partial \operatorname{det} J_{1}}{\partial y^{k}} \psi^{k} J_{1}^{i j} \tilde{p}_{i} \tilde{p}_{j}-\left(\operatorname{det} J_{1}\right)^{-1} \psi^{k} \frac{\partial W^{1}}{\partial y^{k}}
\end{aligned}
$$

with $h$ as in (57). Concerning the function we want to match it with, we first of all need more info about $\Delta_{(n)}$. Using the representation (36) of $J$, we can put

$$
J+a P_{2}=\left(\begin{array}{cc}
J_{1} & 0 \\
J_{21} & 1
\end{array}\right)\left(\begin{array}{cc}
1 & J_{1}^{-1} J_{12} \\
0 & \bar{J}_{2}+a I_{n}
\end{array}\right)
$$

from which it follows that

$$
\sum_{i=1}^{n+1} \Delta_{(i)} a^{i-1}=\operatorname{det}\left(J+a P_{2}\right)=\left(\operatorname{det} J_{1}\right) \operatorname{det}\left(\bar{J}_{2}+a I_{n}\right),
$$

and hence that $\Delta_{(n)}=\left(\operatorname{det} J_{1}\right)\left(\operatorname{tr} \bar{J}_{2}\right)$. It follows from (156) that

$$
\left(\operatorname{det} J_{1}\right)^{-1} H_{(n)}=\frac{1}{2} g^{a b} \tilde{p}_{a} \tilde{p}_{b}+\frac{1}{2}\left(\operatorname{tr} \bar{J}_{2}\right) J^{i j} \tilde{p}_{i} \tilde{p}_{j}+\left(\operatorname{det} J_{1}\right)^{-1} W_{(n)} .
$$

It looks by far not obvious that the expressions (60) and (63) would differ only by one term (up to irrelevant functions of time only). We shall compare them indirectly by computing their derivatives with respect to the $x^{a}$ and $\tilde{p}_{a}$. The latter is easy and gives

$$
\frac{\partial \tilde{h}}{\partial \tilde{p}_{a}}=g^{a b} \tilde{p}_{b}+J^{a i} \tilde{p}_{i}, \quad \frac{\partial}{\partial \tilde{p}_{a}}\left(\left(\operatorname{det} J_{1}\right)^{-1} H_{(n)}\right)=g^{a b} \tilde{p}_{b},
$$

which is in line with the result we want to prove. For the other derivatives, the computations can be written in a somewhat more compact form if we use the basis of vector fields

$$
X_{a}=\frac{\partial}{\partial x^{a}}+\Gamma_{a b}^{c} \tilde{p}_{c} \frac{\partial}{\partial \tilde{p}_{b}}
$$

adapted to the connection, rather than the coordinate derivatives with respect to $x^{a}$. One can verify, recalling that $J_{a}^{k}=\partial \psi^{k} / \partial x^{a}$, that $X_{a}(\tilde{h})$ can be written as

$$
\begin{aligned}
X_{a}(\tilde{h})= & J_{\mid a}^{b i} \tilde{p}_{b} \tilde{p}_{i}+\frac{1}{2}\left(J_{\mid a}^{b i} J_{b}^{j}+J^{b i} J_{b \mid a}^{j}\right) \tilde{p}_{i} \tilde{p}_{j} \\
& +J_{a \mid k}^{i} J_{1}^{j k} \tilde{p}_{i} \tilde{p}_{j}-\frac{1}{2} J_{a}^{k} J_{1 \mid k}^{i j} \tilde{p}_{i} \tilde{p}_{j}-\frac{1}{2}\left(\operatorname{det} J_{1}\right)^{-1} \frac{\partial \operatorname{det} J_{1}}{\partial y^{k}} J_{a}^{k} J_{1}^{i j} \tilde{p}_{i} \tilde{p}_{j} \\
& +\frac{\partial V}{\partial x^{a}}-\left(\operatorname{det} J_{1}\right)^{-1} J_{a}^{k} \frac{\partial W^{1}}{\partial y^{k}} .
\end{aligned}
$$

Making use of the scKt properties of $J$ and $J_{1}$, plus the property (4) for $J_{1}$, this expression considerably simplifies and finally reduces to

$$
\begin{aligned}
X_{a}(\tilde{h})= & \frac{1}{2} \alpha_{k} g^{i k} \tilde{p}_{i} \tilde{p}_{a}-\frac{1}{2} \alpha_{l}\left(J_{1}^{-1}\right)_{k}^{l} J_{a}^{k} J_{1}^{i j} \tilde{p}_{i} \tilde{p}_{j}+\frac{1}{2} \alpha_{a} J_{1}^{i j} \tilde{p}_{i} \tilde{p}_{j} \\
& +\frac{\partial V}{\partial x^{a}}-\left(\operatorname{det} J_{1}\right)^{-1} J_{a}^{k} \frac{\partial W^{1}}{\partial y^{k}},
\end{aligned}
$$


where $\alpha$ as before stands for $d(\operatorname{tr} J)$. Note in passing that, for example, $J^{i j} \equiv J_{1}^{i j}$. The computation of $X_{a}\left(\left(\operatorname{det} J_{1}\right)^{-1} H_{(n)}\right)$ is much easier and gives

$$
X_{a}\left(\left(\operatorname{det} J_{1}\right)^{-1} H_{(n)}\right)=\frac{1}{2} \bar{\alpha}_{a} J_{1}^{i j} \tilde{p}_{i} \tilde{p}_{j}+\left(\operatorname{det} J_{1}\right)^{-1} \frac{\partial W_{(n)}}{\partial x^{a}},
$$

with $\bar{\alpha}=d\left(\operatorname{tr} \bar{J}_{2}\right)$. We need to make three more observations now. The first is that

$$
X_{a}\left(J^{b i} \tilde{p}_{i} \tilde{p}_{b}\right)=J_{\mid a}^{b i} \tilde{p}_{i} \tilde{p}_{b}=\frac{1}{2} \alpha_{k} g^{i k} \tilde{p}_{i} \tilde{p}_{a}
$$

which takes account of the first term of $X_{a}(\tilde{h})$. Secondly, we recall the difference between $\bar{\alpha}_{a}$ and $\alpha_{a}$, as obtained in (39), which makes that the first term of $X_{a}\left(\left(\operatorname{det} J_{1}\right)^{-1} H_{(n)}\right)$ matches two terms of $X_{a}(\tilde{h})$. Finally, the terms not containing momenta also match as a result of (42), taking into account that $\mu_{2}=-d_{2} V$. The conclusion now is that

$$
X_{a}(\tilde{h})=X_{a}\left(\left(\operatorname{det} J_{1}\right)^{-1} H_{(n)}+J^{b i} \tilde{p}_{i} \tilde{p}_{b}\right) .
$$

Remember that the two functions under consideration are regarded here as depending on the $x^{a}, \tilde{p}_{a}$ and time $t$ (along solutions of the driving equations). Since their derivatives with respect to the $x^{a}$ and $\tilde{p}_{a}$ are the same, the conclusion is that they are indeed equal up to an additive function of time.

The idea now is to try to get rid of the second term on the right in (58) by a further, suitable canonical transformation. It is of some interest to look at this kind of question in all generality and to observe that it can be resolved indeed by a suitable point transformation, which necessarily must be time-dependent however. The notations used in discussing this general question below have nothing to do with any of the specific situations encountered so far.

Lemma 3. Suppose that $H_{1}(q, p, t)$ and $H_{2}(q, p, t)$ are two functions which differ by terms linear in the $p_{i}$. Then, there exists a point transformation, $(q, p) \leftrightarrow(Q, P)$ say, such that the transformed Hamiltonian of the system with Hamiltonian $\mathrm{H}_{1}$ becomes the function $\mathrm{H}_{2}$ expressed in the new variables.

Proof. By assumption, we have $H_{1}=H_{2}+\rho^{i}(q, t) p_{i}$ for some functions $\rho^{i}$. If $F(q, P, t)=$ $P_{i} Q^{i}(q, t)$ is the generating function of an as yet unspecified point transformation, we know that the transformed Hamiltonian of the system with Hamiltonian $H_{1}$ will be given by

$$
\tilde{H}_{1}=H_{1}+\frac{\partial F}{\partial t}=H_{2}+\rho^{i} \frac{\partial Q^{j}}{\partial q^{i}} P_{j}+\frac{\partial Q^{j}}{\partial t} P_{j}
$$

so that the desired effect requires that each of the $Q^{j}$ satisfies

$$
\frac{\partial Q^{j}}{\partial t}+\rho^{i} \frac{\partial Q^{j}}{\partial q^{i}}=0 .
$$

In other words, we need $n$ functionally independent first integrals of the equations $\dot{q}^{i}=$ $\rho^{i}(q, t)$, which can be done in principle and is of course the same as saying that we have to integrate those equations. Note that even if the given $\rho^{i}$ would not depend on time, this procedure can only work with a time-dependent canonical transformation. 
In the case of interest, we are looking with equation (58) at linear terms of the form

$$
\rho^{a}(x, t) \tilde{p}_{a}=J^{a i}(x, y(t)) \tilde{p}_{i}(t) \tilde{p}_{a}
$$

We can try to find first integrals of the equations $\dot{x}^{a}=\rho^{a}(x, t)$ of the form $u^{a}=u^{a}(y(t), x)$, i.e. which are such that the time-dependence originates from solutions $y(t)$ of the driving equations. This means that we have $\dot{y}^{k}=J^{k i} \tilde{p}_{i}$, and the first integral condition becomes

$$
\frac{\partial u^{a}}{\partial t}+\rho^{b} \frac{\partial u^{a}}{\partial x^{b}}=\tilde{p}_{i}\left(J^{i k} \frac{\partial u^{a}}{\partial y^{k}}+J^{i b} \frac{\partial u^{a}}{\partial x^{b}}\right)=0 .
$$

We can now prove one of our main results, for which we go back to the supplementary assumption of the end of Section 4. The eigenfunctions $u^{a}(y, x)$ which were introduced there will now be used as new coordinates for the driven system, along solutions $y(t)$ of the driving system, and we will denote the corresponding conjugate momenta (for reference to the notations used in the Euclidean case in [10]) by $s_{a}$.

Proposition 4. Assume that the equation $\operatorname{det}\left(J-\lambda P_{2}\right)=0$ has $n$ functionally independent solutions $u^{a}(y, x)$. Then, the canonical transformation $\left(x^{a}, p_{a}\right) \leftrightarrow\left(x^{a}, \tilde{p}_{a}\right)$ with generating function (54), followed by the canonical transformation $\left(x^{a}, \tilde{p}_{a}\right) \leftrightarrow\left(u^{a}, s_{a}\right)$ with generating function $F(x, s, t)=s_{a} u^{a}(y(t), x)$, has the effect of transforming the Hamiltonian $h$ of the driven system into the function $\left(\operatorname{det} J_{1}\right)^{-1} H_{(n)}$.

Proof. We know from Lemma 2 that the first step brings the Hamiltonian $h$ into the form (58). According to Lemma 3, the second step will eliminate the linear terms in the momenta in (58), provided the functions $u^{a}(y, x)$ have the property of making the right-hand side of (64) vanish. But the $u^{a}$ satisfy the relations (45), from which it follows by a left action of $P_{1}$ that

$$
J_{1} P_{1}\left(d u^{a}\right)+J_{21} P_{2}\left(d u^{a}\right)=0 \quad \text { or } \quad\left(J_{j}^{k} \frac{\partial u^{a}}{\partial y^{k}}+J_{j}^{b} \frac{\partial u^{a}}{\partial x^{b}}\right) d y^{j}=0 .
$$

The desired result now follows by raising an index.

At this point, it is important to be aware of another general and in fact very simple result.

Lemma 4. Assume that a Hamiltonian system has a Hamiltonian $K(q, p, t)$ of the form $K=\gamma(t) H(q, p, t)$, whereby $H$ is a first integral of the system and $\gamma$ is an arbitrary function of $t$ only. Then $H$ in fact cannot explicitly depend on time and the time-dependent Hamilton-Jacobi equation for $K(q, p, t)$ reduces to the autonomous one for $H$.

Proof. It is obvious that (with respect to the standard Poisson bracket) we have $\{K, H\}=\gamma\{H, H\}=0$, so that the property $\dot{H}=0$ reduces to $\partial H / \partial t=0$. The Hamilton-Jacobi equation for $K$ then reads

$$
\gamma(t) H\left(q, \frac{\partial S}{\partial q}\right)+\frac{\partial S}{\partial t}=0
$$


and looking for a complete solution $S\left(q, t, \alpha_{i}\right)$ of the form $S=W\left(q, \alpha_{i}\right)-\alpha_{1} \int \gamma d t$ immediately reduces it to the Hamilton-Jacobi equation

$$
H\left(q, \frac{\partial W}{\partial q}\right)=\alpha_{1}
$$

for the autonomous function $H$.

Since $\operatorname{det} J_{1}$, along solutions of the driving system, is a function of time only and $H_{(n)}$ is known to be a first integral of the driven system under the same circumstances, it is clear that the assumptions of Proposition 4 precisely bring us in a situation where we can draw the quite surprising conclusion that $H_{(n)}$ will no longer be time-dependent in the $(u, s)$ coordinates and separability of the Hamilton-Jacobi equation for the driven system is essentially a matter of separability of $H_{(n)}$. It remains to convince ourselves that the Hamilton-Jacobi equation for a system with $H_{(n)}$ as Hamiltonian is indeed separable. Now comes a rather subtle point in the argumentation. The point is this! We want to test separability of $H_{(n)}$ by using criteria which have an intrinsic, i.e. coordinate independent meaning. As such, it is the function $H_{(n)}$ which matters, expressed in any kind of canonical coordinates. Transformation formulas from one set of coordinates to another can depend on external parameters then, if needed, but should not be regarded as depending on time because time-dependent canonical transformations do more to the Hamiltonian function than just expressing it in the new variables.

To be concrete now, the sufficient conditions for separability which we want to invoke are intrinsic indeed: they comprise the existence of a special conformal Killing tensor $J$ for the kinetic energy metric of the Hamiltonian, plus a corresponding condition for admissible potentials $V$. The condition $d(J d V)=0$ in [2] for example, which actually corresponds to the particular case of the cofactor condition $A \mu=-d W$ when the forces are conservative. It is well known (see e.g. 4]) that if the scKt involved in these conditions has functionally independent eigenfunctions, then these are separation coordinates for the Hamilton-Jacobi equation. It so happens that we have needed these $u^{a}$-coordinates already to prove in Proposition 4 that the original Hamiltonian $h$ of the driven system can be transformed into the function $H_{(n)}$ (up to a factor). But having shown this way that HamiltonJacobi separability becomes a question about the function $H_{(n)}$, it is more appropriate to put this function to the test in the set of canonical coordinates $\left(x^{a}, \tilde{p}_{a}\right)$ which naturally presents itself prior to introducing the separation coordinates. The delicate issue alluded to above is that, when we subsequently want to pass to the new variables $\left(u^{a}, s_{a}\right)$ again, the interpretation for this part of the story is that the functions $u^{a}(y, x)$ are regarded then as depending parametrically on the $y$-coordinates of the driving system (not as a time-dependent transformation along solutions $y(t)$ of that system).

Going back to the expression (63) of $H_{(n)}$, taking into account that the function $H_{(n+1)}$ in (59) is actually a constant parameter (along solutions of the driving system) which we called $E^{1}$ in (15), we have that

$$
H_{(n)}=\frac{1}{2}\left(\operatorname{det} J_{1}\right) g^{a b} \tilde{p}_{a} \tilde{p}_{b}+\left(\operatorname{tr} \bar{J}_{2}\right)\left(E^{1}-W^{1}(y)\right)+W_{(n)}(y, x) .
$$

This is the right expression for activating our test because all the ingredients we need for that have been prepared in Section 4 . 
Proposition 5. $\bar{J}_{2}$ is a special conformal Killing tensor for the metric associated to the quadratic terms in (66) and the remaining terms satisfy the conditions for an admissible potential for Hamilton-Jacobi separability.

Proof. We know from Proposition 1 that $\bar{J}_{2}$ is a scKt for $g_{2}=\left(g_{a b}(x)\right)$ and recall that the characterizing property (2) of scKts, when expressed in terms of the underlying type $(1,1)$ tensor field is given by (12), with $\alpha=d(\operatorname{tr} J)$. It is then clear that the same $J$ is also a scKt with respect to any constant multiple of $g$. In the case of the quadratic terms in (66), we are precisely looking at a constant multiple of the metric $g_{2}$ since $\left(\operatorname{det} J_{1}\right)$ is a function of the external $y$-parameters only, whence the conclusion about $\bar{J}_{2}$. We further observed above that the condition for admissible potentials is a reduced form of the cofactor condition that $(\operatorname{cof} J) \mu$ should be closed. For the situation at hand we already know from Proposition 2 that the function $W_{(n)}$ satisfies this condition with respect to the scKt $\bar{J}_{2}$. It remains to show that the same is true for the remaining term in (66) which in fact, since the factor $\left(E^{1}-W^{1}(y)\right)$ can be treated as a constant here, amounts to saying that the function $\operatorname{tr} \bar{J}_{2}$ satisfies the condition. But this is trivially the case, because it follows from (4) that for any tensor $J$ with vanishing Nijenhuis torsion $(\operatorname{cof} J) d(\operatorname{tr} J)=d(\operatorname{det} J)$.

We sum up the main results about the driven system now. We know since Section 3 that the driven system, along solutions of the driving system, has $n$ integrals $H_{(i)}$ which are in involution with respect to two Poisson structures, one of which is the standard one when using coordinates adapted to the integrable distributions $K$ and $K^{\perp}$. Under the assumption that the characteristic equation $\operatorname{det}\left(J-\lambda P_{2}\right)=0$ has $n$ functionally independent solutions $u^{a}(y, x)$, we have seen in Proposition 4 that the given Hamiltonian $h$ of the driven system, can be transformed into the function $\left(\operatorname{det} J_{1}\right)^{-1} H_{(n)}$. The fact that all the $H_{(i)}$ are first integrals then implies that

$$
\frac{\partial H_{(i)}}{\partial t}+\left(\operatorname{det} J_{1}\right)^{-1}\left\{H_{(i)}, H_{(n)}\right\}=0 .
$$

But this, in view of the involutivity, actually means that none of the integrals will be time-dependent when expressed in the canonical coordinates $\left(u^{a}, s_{a}\right)$. This is in line with the results of Proposition 5 which mainly says that due to the existence of a scKt $\bar{J}_{2}$, the Hamilton-Jacobi equation of the (autonomous) function $H_{(n)}$ is separable. In fact, referring to the results which can be found in [4] for example, we can state more precisely that we are looking at a separable system of Stäckel type and that the eigenfunctions $u^{a}(y, x)$ of $\bar{J}_{2}$ are orthogonal separation coordinates. It should then be true indeed that in those coordinates we have $n$ time-independent quadratic integrals in involution. Note that for the function $H_{(n)}$, for example, this time-independence means among other things (see the expression (66) ) that the function $W_{(n)}-\left(\operatorname{tr} \bar{J}_{2}\right) W^{1}$, when passing from the coordinates $(y, x)$ to the coordinates $(y, u(y, x))$, should become independent of the $y$-variables.

To give some further backing for all these rather subtle properties, we carry out more explicit calculations in the Appendix. The programme there is that we complete the recursive scheme started in Section 3 by proving, in analogy with the expressions (22, 23), explicit results for the different parts of all the $A_{(i)}$ tensors and computing explicit expressions for the corresponding functions $H_{(i)}$. 


\section{Examples}

For examples which exhibit all features of our generalization, we probably should think of systems with at least three degrees of freedom and a non-Euclidean metric. But going into the details of such applications would substantially add to the length of this already rather long paper, so we will keep this for a separate study and limit ourselves here to simpler two-dimensional situations. The first example is inspired by an integrability study in [17] on what were called 'generalized Hénon-Heiles systems', which are SODEs of the form

$$
\begin{aligned}
& \ddot{q}_{1}=-c_{1} q_{1}+b q_{1}^{2}-a q_{2}^{2}, \\
& \ddot{q}_{2}=-c_{2} q_{2}-2 m q_{1} q_{2} .
\end{aligned}
$$

Compared to older case studies of integrability of Hénon-Heiles systems, the generalization comes from the extra parameters $a$ and $m$, which are motivated by allowing a Lagrangian description of the system in which the Hessian of the Lagrangian need not be (a constant multiple of) the unit matrix. The investigation carried out in [17] mainly consisted in looking for all possible parameter cases for which the system has two independent quadratic integrals. It led to the identification of three new cases, which are in some sense degenerate cases, because either $a$ or $m$ is zero, meaning that the Lagrangian one originally thought of is degenerate. As a result, there is no corresponding Hamiltonian which in the standard cases is always available as the first of two integrals in involution. A subsidiary question then was: to what extent can the two integrals in those degenerate cases be understood as being in involution, and it was argued that this can be resolved by constructing, in principle, a suitably adapted non-standard Poisson structure. As the equations in the case that either $a$ or $m$ is zero clearly exhibit partial decoupling, there is a good chance that those degenerate cases actually fit within our present theory, which is what we will discuss now.

Consider the case where $m=0$ and $b=0$, so that the system reduces to

$$
\begin{aligned}
& \ddot{q}_{1}=-c_{1} q_{1}-a q_{2}^{2}, \\
& \ddot{q}_{2}=-c_{2} q_{2} .
\end{aligned}
$$

These equations are of course easy to solve without further ado, but they must serve here in the first place to illustrate various aspects of our theory. The second equation plays the role of driving equation. For consistency with the notations in the preceding sections we rename the variables as $q_{2}=y, q_{1}=x$ and write

$$
\begin{aligned}
& \ddot{y}=-c_{2} y, \\
& \ddot{x}=-c_{1} x-a y^{2} .
\end{aligned}
$$

Putting $p_{y}=\dot{y}$ and $p_{x}=\dot{x}$ the two quadratic integrals read

$$
\begin{aligned}
F_{2}= & \frac{1}{2} p_{y}^{2}+ \\
F_{1}= & \frac{1}{2} c_{2} y^{2} \\
& \\
& +a\left(c_{1}-4 c_{2}\right) p_{x}^{2}+2 a y p_{x} p_{y}-2 a x y_{y}^{2}+\frac{1}{2} c_{1} a^{2} y^{4} .
\end{aligned}
$$


Obviously $F_{2}$ is a Hamiltonian for the driving equation and is likely to be identifiable with the function $H_{(2)}$ in the theory (see (27) knowing that $n=1$ here). The idea now is the following. Since $F_{1}$ is a first integral of the complete system, its quadratic part identifies a Killing tensor $A$. Looking at the tensor $J$ of which $A$ is the cofactor, this may or may not be a scKt in general. It was shown in [9] however that this will always be the case for the Euclidean metric in dimension 2. If the component $J_{1}$ is nonsingular therefore, we must be in a situation covered by our present theory and all the features we discussed should apply, with $F_{1}=H_{(1)}=H$, the Hamiltonian of the quasi-Hamiltonian representation (24). The extra assumption that the driven system should have a genuine potential, parametrically depending on the $y$-variables, is also automatically satisfied here by dimension.

From the expression of $F_{1}$, we see that

$$
A=\left(\begin{array}{cc}
-4 a x & 2 a y \\
2 a y & c_{1}-4 c_{2}
\end{array}\right) .
$$

and $A=\operatorname{cof} J$, where $J$ is the tensor with the following matrix components

$$
J=\left(\begin{array}{cc}
c_{1}-4 c_{2} & -2 a y \\
-2 a y & -4 a x
\end{array}\right)
$$

This tensor is indeed a scKt with respect to the Euclidean metric, and its $J_{1}$ component is nonsingular (assuming $c_{1} \neq 4 c_{2}$ ), so we are in business and $A^{1}=\operatorname{cof} J_{1}=1$. For completeness, observe that

$$
\mu=-c_{2} y d y-\left(c_{1} x+a y^{2}\right) d x .
$$

in this example and that $A \mu=-d W$ indeed, with

$$
W=\frac{1}{2} c_{1}\left(c_{1}-4 c_{2}\right) x^{2}+a\left(c_{1}-2 c_{2}\right) x y^{2}+\frac{1}{2} a^{2} y^{4} .
$$

The function $h$ for the standard Hamiltonian representation of the driven equation (with parameter $y$ ) is given by

$$
h=\frac{1}{2} p_{x}^{2}+a y^{2} x+\frac{1}{2} c_{1} x^{2} .
$$

Let us now further illustrate the subtleties of the theory, as explained in Sections 4 to 6 . We start by computing the function $\tilde{h}$ as defined in (55). The tensor $\bar{J}_{2}$ here reads

$$
\bar{J}_{2}=-\left(4 a x+\frac{4 a^{2} y^{2}}{c_{1}-4 c_{2}}\right) \frac{\partial}{\partial x} \otimes d x .
$$

and the new momenta defined in $(52,53)$ become

$$
\begin{aligned}
& p_{y}=\left(c_{1}-4 c_{2}\right) \tilde{p}_{y}, \\
& p_{x}=\tilde{p}_{x}-2 a y \tilde{p}_{y} .
\end{aligned}
$$

In those new variables, the expression for $F_{1}$ becomes

$$
\begin{gathered}
F_{1}=\frac{1}{2}\left(c_{1}-4 c_{2}\right) \tilde{p}_{x}^{2}-2 a^{2} y^{2}\left(c_{1}-4 c_{2}\right) \tilde{p}_{y}^{2}-2 a x\left(c_{1}-4 c_{2}\right)^{2} \tilde{p}_{y}^{2} \\
+\frac{1}{2} c_{1}\left(c_{1}-4 c_{2}\right) x^{2}+a\left(c_{1}-2 c_{2}\right) x y^{2}+\frac{1}{2} a^{2} y^{4}
\end{gathered}
$$


which is in agreement with (63). For the computation of $\tilde{h}$ on the other hand, we need the generating function (54) of the time-dependent canonical transformation $\left(x, p_{x}\right) \leftrightarrow\left(x, \tilde{p}_{x}\right)$. We see from (68) that $J_{2}^{1}=-2 a y=\partial \psi / \partial x$ with $\psi=-2 a x y$. The generating function $F$ thus reads

$$
F\left(x, \tilde{p}_{x}, t\right)=x \tilde{p}_{x}-2 a x y(t) \tilde{p}_{y}(t),
$$

and computing its partial time derivative involves making use of the driving equation. The resulting expression for $\tilde{h}$ is found to be

$$
\tilde{h}=\frac{1}{2} \tilde{p}_{x}^{2}+2 a^{2} y^{2} \tilde{p}_{y}^{2}-2 a x\left(c_{1}-4 c_{2}\right) \tilde{p}_{y}^{2}-2 a y \tilde{p}_{x} \tilde{p}_{y}+a y^{2} x+\frac{1}{2} c_{1} x^{2}+\frac{2 a y^{2} x c_{2}}{c_{1}-4 c_{2}} .
$$

One can verify that $\tilde{h}$ and $H_{(1)}=F_{1}$ indeed verify the requirement (58) of Lemma 2 to within an additive function of time, which is the function

$$
4 a^{2} y^{2} \tilde{p}_{y}^{2}-\frac{a^{2} y^{4}}{2\left(c_{1}-4 c_{2}\right)}
$$

and of course can be ignored in writing down Hamilton's equations. For the final canonical transformation to be applied to $\tilde{h}$, we need the eigenfunction $u(y, x)$ of $\bar{J}_{2}$, which is found to be (see (69) $)$

$$
u(y, x)=-\frac{4 a^{2} y^{2}}{c_{1}-4 c_{2}}-4 a x .
$$

The time-dependent canonical transformation with generating function $F(x, s, t)=s u(y(t), x)$ now transforms the relevant part of $\tilde{h}$ into the function

$$
\left(\operatorname{det} J_{1}\right)^{-1} F_{1}=8 a^{2} s^{2}+\frac{1}{2} u\left(c_{1}-4 c_{2}\right) \tilde{p}_{y}^{2}+\frac{1}{2} u \frac{c_{2}}{c_{1}-4 c_{2}} y^{2}+\frac{c_{1}}{32 a^{2}} u^{2} .
$$

It then easily follows by taking into account that $F_{2}$ is a constant along solutions of the driving equation, that

$$
F_{1}=8\left(c_{1}-4 c_{2}\right) a^{2} s^{2}+u F_{2}+\frac{c_{1}\left(c_{1}-4 c_{2}\right)}{32 a^{2}} u^{2}
$$

which indeed no longer depends explicitly on time.

The other two degenerate cases in [17] also fit within the present theory to some extent, but are even more peculiar. One can verify that the two integrals in those cases again can be understood by the fact that the system is a driven cofactor system. This also explains why the equations partially decouple. But it so happens that the $J_{1}$ part of the scKt $J$ in those cases is zero, so that we are not in the generic case of a nonsingular $J_{1}$, which was the assumption in the preceding sections.

With a second simple example, we want to illustrate and test mainly the beginning of our theory, before suitable coordinates for partial decoupling have been identified, i.e. the situation covered by the conditions of Definition 2. Consider the system

$$
\begin{aligned}
& \ddot{q}_{1}=5 q_{1}-4 q_{2}, \\
& \ddot{q}_{2}=q_{1} .
\end{aligned}
$$


The connection coefficients of the SODE connection are all zero and the $(1,1)$ tensor $\Phi$ has the following matrix representation

$$
\left(\Phi_{j}^{i}\right)=\left(-\frac{\partial f^{i}}{\partial q^{j}}\right)=\left(\begin{array}{ll}
-5 & 4 \\
-1 & 0
\end{array}\right) .
$$

With such a simple, constant Jacobi endomorphism, finding a distribution $K$ which entails submersiveness of the system, i.e. which satisfies the conditions (5) of Definition 2, is simply a matter of looking for a 1-dimensional eigenspace of $\Phi$. We choose

$$
K=\operatorname{sp}\left\{\frac{\partial}{\partial q_{1}}+\frac{\partial}{\partial q_{2}}\right\} .
$$

Note that submersiveness now is ensured and is not related to the existence of a cofactor representation of our system. The problem of detecting such a representation is quite interesting in its own right. It bears to some extent resemblance to the inverse problem of the calculus of variations, because there is a certain freedom in selecting a multiplier matrix $g$ first. For example, making the obvious choice of the unit matrix for $g$, or expressed in more mechanical terms, associating the given equations with a the standard kinetic energy $T=\frac{1}{2}\left(\dot{q}_{1}^{2}+\dot{q}_{2}^{2}\right)$ will not work! Indeed, one can verify that with this $g$, there is no special conformal Killing tensor $J$ for which the right-hand sides of the equations will satisfy the condition $D_{J} \mu=0$. On the other hand, the following choice for $g$ turns out to be appropriate. Take

$$
\left(g_{i j}\right)=\left(\begin{array}{cc}
1 & -1 \\
-1 & 10
\end{array}\right)
$$

then, a scKt $J$ with respect to $g$ is easily found to be

$$
\left(J_{i j}\right)=\left(\begin{array}{cc}
2 q_{1}-2 q_{2} & q_{1}+8 q_{2} \\
q_{1}+8 q_{2} & -4 q_{1}+40 q_{2}
\end{array}\right) .
$$

But more importantly, the nonconservative forces of our system are now of the appropriate form. That is to say, multiplying the right-hand sides of the equations with $g$, the 1 -form $\mu$ is found to be

$$
\mu=4\left(q_{1}-q_{2}\right) d q_{1}+\left(5 q_{1}+4 q_{2}\right) d q_{2},
$$

and computing the cofactor tensor $A$ of $J$, one can verify that $A \mu$ indeed is closed (or equivalently $\left.D_{J} \mu=0\right)$. We can now compute the orthogonal complement of the distribution $K$ and see whether the final conditions for a driven cofactor system are verified. Since $g_{11}+g_{21}=0$, it easily follows that

$$
K^{\perp}=\operatorname{sp}\left\{\frac{\partial}{\partial q_{1}}\right\}
$$

We have

$$
\mathrm{D}^{H} \mu=4 d q_{1} \otimes d q_{1}+5 d q_{1} \otimes d q_{2}-4 d q_{2} \otimes d q_{1}+4 d q_{2} \otimes d q_{2} .
$$

It is clear then that $\mathrm{D}^{H} \mu\left(K^{\perp}, K\right) \neq 0$, whereas obviously $d \mu(K, K)=0$ by dimension. Hence, all requirements of Definition 2 are met. Integrating the distributions $K$ and $K^{\perp}$, 
suitable coordinates for the decoupling are found to be $x=q_{2}, y=q_{1}-q_{2}$ and the transformed system becomes

$$
\begin{aligned}
& \ddot{y}=4 y, \\
& \ddot{x}=x+y .
\end{aligned}
$$

We leave it to the reader to verify from here, exactly as we did with the first example, that all the other features of our theory hold true, in particular those related to the consecutive canonical transformations relating the Hamiltonian $h$ of the driven system to the quadratic integral $H_{(1)}$.

\section{Appendix}

Consider the recursive scheme for the tensors $A_{(i)}$, in particular the relations (18) for $i=1, \ldots, n$. We have seen that taking $i=n$ led to determining relations for three of the four parts of $A_{(n)}$ (see (22) ), while the remaining block $A_{(n) 1}$ had to be obtained from taking subsequently $i=n-1$. This procedure can be continued all the way down, and the essential features of the recursion are captured in the following statement.

Proposition 6. Suppose that we know the tensor parts $A_{(i+1) 21}, A_{(i+1) 12}$ and $A_{(i+1) 2}$ and that they satisfy the identities

$$
\begin{aligned}
& J_{1} A_{(i+1) 12}+J_{12} A_{(i+1) 2} \equiv 0 \equiv A_{(i+1) 21} J_{1}+A_{(i+1) 2} J_{21} \\
& J_{21} A_{(i+1) 12}+J_{2} A_{(i+1) 2} \equiv A_{(i+1) 21} J_{12}+A_{(i+1) 2} J_{2} .
\end{aligned}
$$

Then the following are determining equations for the completion of the construction of $A_{(i+1)}$ and for the next step in the recursion

$$
\begin{aligned}
A_{(i+1) 1} & =\Delta_{(i+1)} J_{1}^{-1}-J_{1}^{-1} J_{12} A_{(i+1) 21} \\
A_{(i) 21} & =-J_{21} A_{(i+1) 1}-J_{2} A_{(i+1) 21} \\
A_{(i) 12} & =-A_{(i+1) 1} J_{12}-A_{(i+1) 12} J_{2} \\
A_{(i) 2} & =\Delta_{(i+1)} P_{2}-J_{21} A_{(i+1) 12}-J_{2} A_{(i+1) 2} .
\end{aligned}
$$

Moreover, the newly obtained parts of $A_{(i)}$ will satisfy the same three identities as those assumed for $A_{(i+1)}$, while the completion of $A_{(i+1)}$ will give rise to the following supplementary identity:

$$
J_{1} A_{(i+1) 1}+J_{12} A_{(i+1) 21} \equiv A_{(i+1) 1} J_{1}+A_{(i+1) 12} J_{21}
$$

ProOf. Consider the recursion relation (18) which in fact has two parts. The idea is to compose each of those parts on the left and on the right with one of the projectors $P_{i}$. This gives rise to a total of 8 equations. For example, acting with $P_{1}$ on both sides of the relation $J A_{(i+1)}+P_{2} A_{(i)}=\Delta_{(i+1)} I_{N}$ (which we can call a $\left(P_{1}, P_{1}\right)$ action for brevity) implies that we must have

$$
J_{1} A_{(i+1) 1}+J_{12} A_{(i+1) 21}=\Delta_{(i+1)} P_{1},
$$


from which the determining equation (72) follows. Likewise, a two-sided $\left(P_{2}, P_{1}\right)$ action will generate (73) and the double $P_{2}$ action generates (75). The remaining $\left(P_{1}, P_{2}\right)$ combination merely confirms the first of the assumed identities (70). Starting from the other part in (18), the determining equation (74) will follow from a $\left(P_{1}, P_{2}\right)$ action. The $\left(P_{2}, P_{1}\right)$ combination confirms the second of the assumed identities in (70). The double $P_{2}$ action gives rise to another determining equation for $A_{(i) 2}$, which is consistent with the first one in view of the identity (71). Finally the double $P_{1}$ action implies that, again for consistency, $A_{(i+1)}$ must further satisfy the identity (76) $)$. It is then a straightforward computation to verify that the obtained blocks $A_{(i) 21}, A_{(i) 12}$ and $A_{(i) 2}$ will satisfy corresponding identities of the form (70) and (71) in view of those assumed for $A_{(i+1)}$. This concludes the full recursion step.

The identities which the different parts of all $A_{(i)}$ tensors satisfy are important to get to a considerable simplification of the recursive scheme. Indeed, as will be shown now, it turns out that knowledge of the block $A_{(i) 2}$ suffices to determine the three other blocks of $A_{(i)}$. Moreover we can set up a recursive scheme to determine $A_{(i) 2}$ from $A_{(i+1) 2}$ and we shall see that this procedure brings the tensor $\bar{J}_{2}$ back into the spotlights.

It follows from the identities (70) that for each $i=1, \ldots, n$ :

$$
\begin{aligned}
& A_{(i) 12}=-J_{1}^{-1} J_{12} A_{(i) 2}, \\
& A_{(i) 21}=-A_{(i) 2} J_{21} J_{1}^{-1},
\end{aligned}
$$

and subsequently from the defining relation (72) that

$$
A_{(i) 1}=\Delta_{(i)} J_{1}^{-1}+J_{1}^{-1} J_{12} A_{(i) 2} J_{21} J_{1}^{-1} .
$$

Making use of (77, 78) in the identity (71) it is easy to see that this expresses the commutativity

$$
\bar{J}_{2} A_{(i) 2}=A_{(i) 2} \bar{J}_{2} .
$$

Finally, the determining equation (75) reduces to

$$
A_{(i) 2}=\Delta_{(i+1)} P_{2}-\bar{J}_{2} A_{(i+1) 2} .
$$

Lemma 5. The blocks $A_{(i) 2}=P_{2} \circ A_{(i)} \circ P_{2}$ of the $A_{(i)}$ tensors which determine the quadratic part of the first integrals $H_{(i)}$ are recursively given by

$$
A_{(i) 2}=\Delta_{(i+1)} P_{2}+\sum_{j=1}^{n-i}(-1)^{j} \Delta_{(j+i+1)} \bar{J}_{2}^{j}, \quad i=1, \ldots, n-1,
$$

and all other parts of $A_{(i)}$ follow from $A_{(i) 2}$.

ProOF. We know that $A_{(n) 2}=\Delta_{(n+1)} P_{2}$, with $\Delta_{(n+1)}=\operatorname{det} J_{1}$. The recursive relation (82) then easily follows from (81) by induction. The last part of the statement has already been proved above.

Obviously, we are now in a position to venture computing a more explicit expression for the functions $H_{(i)}$ and it turns out that it is most appropriate to do this in terms of the momenta $\tilde{p}$ again. 
Proposition 7. The quadratic integrals $H_{(i)}($ for $i=1, \ldots, n)$ are given by

$$
H_{(i)}=\frac{1}{2} A_{(i)}^{a b} \tilde{p}_{a} \tilde{p}_{b}+\frac{1}{2} \Delta_{(i)} J_{1}^{k l} \tilde{p}_{k} \tilde{p}_{l}+W_{(i)},
$$

where

$$
A_{(i)}^{a b}=\Delta_{(i+1)} g^{a b}+\sum_{j=1}^{n-i}(-1)^{j} \Delta_{(j+i+1)}\left(\bar{J}_{2}^{j}\right)^{a b} .
$$

Proof. Recall that $H_{(n)}$ has already been computed (see (56)) and is indeed of the form (83). From (26), we further have that

$$
H_{(i)}=\frac{1}{2} A_{(i)}^{a b} p_{a} p_{b}+A_{(i)}^{a k} p_{a} p_{k}+\frac{1}{2} A_{(i)}^{k l} p_{k} p_{l}+W_{(i)} .
$$

Observe first that raising indices in (81) gives rise to the formula

$$
A_{(i)}^{a b}=\Delta_{(i+1)} g^{a b}-\bar{J}_{2 d}^{b} A_{(i+1)}^{d a},
$$

and it subsequently follows from (77) and (79) that

$$
\begin{aligned}
& A_{(i)}^{a k}=J_{1}^{-1}{ }_{l}^{k} J_{b}^{l}\left(\bar{J}_{2}^{b} A_{(i+1)}^{d a}-\Delta_{(i+1)} g^{a b}\right) \\
& A_{(i)}^{k l}=\Delta_{(i)} J_{1}^{-1{ }^{k l}}-{J_{1}^{-1}{ }_{j} l}^{k} J_{b}^{j} \bar{J}_{2 d}^{b} A_{(i+1)}{ }_{c}^{d} J_{m}^{c} J_{1}^{-1^{m k}}+\Delta_{(i+1)} J_{1}^{-1}{ }_{j}^{l} J_{c}^{j} J_{m}^{c} J_{1}^{-1^{m k}} .
\end{aligned}
$$

We now compute each of the quadratic parts of $H_{(i)}$ in terms of the $\tilde{p}$, using $(52,53)$. The first term, for example, becomes

$$
\begin{aligned}
\frac{1}{2} A_{(i)}^{a b} p_{a} p_{b}= & \frac{1}{2} A_{(i)}^{a b} \tilde{p}_{a} \tilde{p}_{b}+\Delta_{(i+1)} J^{b j} \tilde{p}_{b} \tilde{p}_{j}-\bar{J}_{2 d}^{b} A_{(i+1)}^{d a} J_{b}^{j} \tilde{p}_{a} \tilde{p}_{j} \\
& +\frac{1}{2} \Delta_{(i+1)} J^{b j} J_{b}^{k} \tilde{p}_{j} \tilde{p}_{k}-\frac{1}{2} \bar{J}_{2_{d}^{b}} A_{(i+1)}^{d a} J_{a}^{j} J_{b}^{k} \tilde{p}_{j} \tilde{p}_{k} .
\end{aligned}
$$

It so happens that only the first term on the right in this expression survives, in other words all the other terms in the end cancel out when we proceed in the same way with the two other quadratic terms of $H_{(i)}$. The formula (83) then readily follows, while (84) merely is the contravariant form of (82), and will turn out to be useful further on.

As was the case with $H_{(n)}$, we recognize in the expression for the other $H_{(i)}$ part of the constant $E^{1}$ of the driving system. Explicitly, with the help of (15), it is clear that along solutions of the driving system, the $H_{(i)}$ can be written as

$$
H_{(i)}=\frac{1}{2} A_{(i)}^{a b} \tilde{p}_{a} \tilde{p}_{b}+\frac{\Delta_{(i)}}{\operatorname{det} J_{1}}\left(E^{1}-W^{1}\right)+W_{(i)},
$$

whereby we recall that $\operatorname{det} J_{1}=\Delta_{(n+1)}$. We shall now finally illustrate that applying the parameter-dependent coordinate change $\left(x^{a}, \tilde{p}_{a}\right) \leftrightarrow\left(u^{a}(x, y), s_{a}\right)$ turns the expressions for the $H_{(i)}$ into functions which no longer depend on the parameters $y^{i}$, and hence, in their interpretation of first integrals of the driven system along solutions of the driving system, become effectively time-independent quadratic integrals.

According to Proposition 3 , the $u^{a}$ are eigenfunctions of $\bar{J}_{2}$, so that the coordinate expression of $\bar{J}_{2}$, in the variables $(y, u)$ takes the simple form

$$
\bar{J}_{2}=u^{a} \frac{\partial}{\partial u^{a}} \otimes d u^{a} .
$$


Going back to the result (62), it follows that

$$
\sum_{i=1}^{n+1} \Delta_{(i)} a^{i-1}=\left(\operatorname{det} J_{1}\right) \operatorname{det}\left(\bar{J}_{2}+a I_{n}\right)=\left(\operatorname{det} J_{1}\right) \prod_{b=1}^{n}\left(u^{b}+a\right)
$$

which in turn implies that

$$
\Delta_{(i+1)}=\left(\operatorname{det} J_{1}\right) \sigma_{n-i}(u), \quad i=0, \ldots, n,
$$

where $\sigma_{j}(u)$ denotes the elementary symmetric functions.

For information about the functional dependence of the other terms in the potential part of the $H_{(i)}$ we go back to the double cofactor representation of the overall system, which was the start of the recursive scheme in Section 3. We know that with $A(a)$ representing the cofactor tensor of $J+a P_{2}$, the force terms $\mu$ of the overall system satisfy the relation $A(a) \mu=-d W(a)$, or equivalently

$$
\operatorname{det}\left(J+a P_{2}\right) \mu=-\left(J+a P_{2}\right) d W(a) .
$$

Projecting this relation under $P_{1}$ it follows that

$$
\operatorname{det}\left(J+a P_{2}\right) \mu_{1}=-J_{1} P_{1}(d W(a))-J_{21} P_{2}(d W(a))
$$

It is straightforward to compute that under the transformation $(y, x) \leftrightarrow(y, u(x, y))$ the tensors involved acquire the following coordinate expression

$$
P_{1}=\left(\frac{\partial}{\partial y^{i}}+\frac{\partial u^{b}}{\partial y^{i}} \frac{\partial}{\partial u^{b}}\right) \otimes d y^{i}, \quad P_{2}=\frac{\partial}{\partial u^{a}} \otimes d u^{a}-\frac{\partial u^{a}}{\partial y^{j}} \frac{\partial}{\partial u^{a}} \otimes d y^{j},
$$

and

$$
J_{1}=J_{j}^{i}\left(\frac{\partial}{\partial y^{i}}+\frac{\partial u^{b}}{\partial y^{i}} \frac{\partial}{\partial u^{b}}\right) \otimes d y^{j}, \quad J_{21}=J_{i}^{a} \frac{\partial u^{c}}{\partial x^{a}} \frac{\partial}{\partial u^{c}} \otimes d y^{i} .
$$

Concerning the left-hand side of (87) we have to remember also that $\left(\operatorname{det} J_{1}\right) \mu_{1}=-d W^{1}$, since the driving system is of cofactor type with scKt $J_{1}$. It then readily follows, using the property (65), that the coordinate expression of (87) reduces to

$$
\operatorname{det}\left(\bar{J}_{2}+a P_{2}\right) \frac{\partial W^{1}}{\partial y^{k}}=\frac{\partial W(a)}{\partial y^{k}}
$$

and this for all $a$. The expansion (62) thus implies that the functions $W_{(i)}-\left(\Delta_{(i)} / \operatorname{det} J_{1}\right) W^{1}$ do not depend on the $y$ parameters, which together with (86) confirms our objective for the potential part in $H_{(i)}$.

It remains to look at the terms quadratic in the momenta. Expressed in the momenta associated to the $u$-variables, they become

$$
\frac{1}{2} A_{(i)}^{a b} \frac{\partial u^{c}}{\partial x^{a}} \frac{\partial u^{d}}{\partial x^{b}} s_{c} s_{d}
$$


We know that in particular $A_{(n)}^{a b}=\left(\operatorname{det} J_{1}\right) g^{a b}(\mathrm{cf}$. (56) $)$. Since $\bar{J}_{2}$ is a scKt with respect to $g_{2}$, it is symmetric in its covariant or contravariant representation. But $\bar{J}_{2}$ is diagonal in the $u$ coordinates, hence the same is true for the transformed $g_{2}$. Let us rely here further on the fact that we proved by indirect means, mainly as a result of the simple Lemma 4, that $H_{(n)}$ will be time-independent when expressed in the $(u, s)$ variables. The net conclusion then is that the diagonal elements of the transformed $g_{2}$ must be the product of $\left(\operatorname{det} J_{1}\right)^{-1}(y)$ with a function depending on the $u$ variables only. It subsequently follows from the explicit expression of $A_{(i)}^{a b}$ in (84) and the fact that the ratios $\Delta_{(i)} / \operatorname{det} J_{1}$ also are functions of the $u$-variables only (see (86)), that the quadratic part of all $H_{(i)}$ will indeed become time-independent as well, when these functions are looked at as first integrals of the driven system along solutions of the driving system.

To complete the picture it is perhaps worth repeating that the final $H_{(1)}$ in that hierarchy is in fact the Hamiltonian $H$ in the quasi-Hamiltonian representation of the full system and that $\left(\operatorname{det} J_{1}\right)^{-1} A_{(1) 2}$ is the cofactor of $\bar{J}_{2}$. Acting with $\bar{J}_{2}$ on the expression (82) for $i=1$, we thus obtain a polynomial expression satisfied by $\bar{J}_{2}$. It is then comforting for the internal consistency of our results that one can verify that this identity indeed expresses the Cayley-Hamilton theorem applied to $\bar{J}_{2}$.

\section{Acknowledgements}

This work is part of the IRSES project GEOMECH (nr. 246981) within the 7th European Community Framework Programme. We are indebted to the referees for constructive comments and suggestions.

\section{References}

[1] S. Benenti, Inertia tensors and Stäckel systems in the Euclidean spaces, Rend. del Sem. Mat. Torino 50 (1992) 315-341.

[2] S. Benenti, Intrinsic characterization of the variable separation in the Hamilton-Jacobi equation, $J$. Math. Phys. 38 (1997) 6578-6602.

[3] S. Benenti, Special symmetric two-tensors, equivalent dynamical systems, cofactor and bi-cofactor systems, Acta Appl. Math. 87 (2005) 33-91.

[4] M. Crampin, Conformal Killing tensors with vanishing torsion and the separation of variables in the Hamilton-Jacobi equation, Diff. Geom. Appl. 18 (2002) 87-102.

[5] M. Crampin and W. Sarlet, A class of nonconservative lagrangian systems on Riemannian manifolds, J. Math. Phys. 42 (2001) 4313-4326.

[6] M. Crampin, W. Sarlet and G. Thompson, Bi-differential calculi, bi-Hamiltonian systems and conformal Killing tensors, J. Phys. A: Math. Gen. 33 (2000) 8755-8770.

[7] A. Frölicher and A. Nijenhuis, Theory of vector-valued differential forms, Proc. Ned. Acad. Wetensch. Ser. A 59 (1956) 338-359.

[8] M. Kossowski and G. Thompson, Submersive second-order differential equations, Math. Proc. Camb. Phil. Soc. 110 (1991) 207-224. 
[9] H. Lundmark, Higher-dimensional integrable Newton systems with quadratic integrals of motion, Studies in Appl. Math. 110 (2003) 257-296.

[10] H. Lundmark and S. Rauch-Wojciechowski, Driven Newton equations and separable time-dependent potentials, J. Math. Phys. 43 (2002) 6166-6194.

[11] K. Marciniak and M. Błaszak, Non-Hamiltonian systems separable by Hamilton-Jacobi method, $J$. Geom. Phys. 58 (2008) 557575.

[12] E. Martínez, Parallel transport and decoupling, In: Applied differential geometry and mechanics, Volume in honour of the 60th birthday of Michael Crampin, W. Sarlet \& F. Cantrijn (eds.) (Academia Press, Gent) (2003) 83-93.

[13] E. Martínez, J.F. Cariñena and W. Sarlet, Derivations of differential forms along the tangent bundle projection, Diff. Geometry and its Applications 2 (1992) 17-43.

[14] E. Martínez, J.F. Cariñena and W. Sarlet, Derivations of differential forms along the tangent bundle projection II, Diff. Geometry and its Applications 3 (1993) 1-29.

[15] E. Martínez, J.F. Cariñena and W. Sarlet, Geometric characterization of separable second-order equations, Math. Proc. Camb. Phil. Soc. 113 (1993) 205-224.

[16] S. Rauch-Wojciechowski, K. Marciniak and H. Lundmark, Quasi-Lagrangian systems of Newton equations, J. Math. Phys. 40 (1999) 6366-6398.

[17] W. Sarlet, New aspects of integrability of generalized Hénon-Heiles systems, J. Phys. A: Math. Theor. 24 (1991) 5245-5251.

[18] W. Sarlet and W. Vanbiervliet, Geometric characterization of driven cofactor systems, J. Phys. A: Math. Theor. 41 (2008) 042001 (10pp). 